\title{
A behavioural gap in survival beliefs*
}

\author{
Giovanna Apicella $^{\dagger} \quad$ Enrico De Giorgi ${ }^{\ddagger}$
}

April 7, 2021

\begin{abstract}
Life span uncertainty (longevity risk) impacts several economic decisions. Individuals can form and revise their survival beliefs making use of behavioural heuristics. We propose a model of sentiment, in which individuals are assumed to switch between optimistic and pessimistic expectations on their health. When optimism is persistent in the face of health shocks, or when individuals are more likely to change their sentiment from pessimistic to optimistic than otherwise, our model predicts survival under-estimation at young ages and over-estimation at old ages. An empirical analysis based on the longitudinal data from the Health and Retirement Study (HRS) validates our model.
\end{abstract}

Keywords: longevity risk, mortality risk, subjective beliefs, sentiment. JEL Classification: D15, H31.

\section{Introduction}

Forward-looking decisions require that decision-makers form some beliefs about the probabilities of relevant future events. Uncertain residual lifetime, i.e., longevity, is pertinent to a wide range of crucial economic decisions for the individuals, e.g., savings, asset allocation, retirement planning, life insurance, and annuity purchases. In the latter respect, insurance is one of the most important activities in a globalized economic environment. It provides a key risk management tool for households, as well as for companies and governments (Organization for Economic Cooperation and Development 2019). As an example, Bernheim et al. (2003) show that life insurance with adequate coverage would reduce

\footnotetext{
${ }^{*}$ We thank seminar participants at the Politecnico of Milan and at the University of St.Gallen, and participants at the "23rd International Congress on Insurance: Mathematics and Economics" and at the "43rd Annual Meeting of the Italian Association for Mathematics Applied to Economic and Social Sciences" for their helpful comments. We gratefully acknowledge financial support of the Swiss National Science Foundation (SNFS) through grant number 189093.

${ }^{\dagger}$ Department of Economics, School of Economics and Political Science, University of St. Gallen. Email: giovanna.apicella@unisg.ch.

${ }_{\ddagger}$ Department of Economics, School of Economics and Political Science, University of St. Gallen, Bodanstrasse 6, 9000 St. Gallen, Switzerland. Email: enrico.degiorgi@unisg.ch (corresponding author).
} 
poverty rates among surviving wives from $16.3 \%$ (no insurance) to $9.2 \%$. Moreover, the propagation of insurance in developing countries, in particular of life insurance, has a positive effect on economic growth (Sawadogo, Guérineau, and Ouedrago 2018).

Individuals form subjective beliefs about the risky outcome represented by uncertain life duration, with implications on the economic decisions they take (see Puri and Robinson 2007, Post and Hanewald 2013, Previtero 2014, and Heimer, Myrseth, and Schoenle 2019). Hamermesh (1985), Hurd and McGarry (2002), and Perozek (2008) show that subjective beliefs on survival differ from actuarial estimates. Specifically, Hamermesh (1985) reports evidence that people extrapolate changing life tables when they determine their subjective estimates, but the resulting subjective distribution is flatter and has greater variance than its actuarial counterpart. Similarly, Hurd and McGarry (2002) and Perozek (2008) document a gap in survival beliefs with younger (older) individuals underestimating (overestimating) survival compared to the actuarial counterpart. Perozek (2008) also illustrates a gender gap, as in their sample female participants tend to underestimate survival, while male participants tend to overestimate it.

There are currently different views in the literature on whether the gap between subjective and actuarial probabilities is due to psychological biases. On the one hand, Hurd and McGarry (2002) and Perozek (2008) show that subjective probabilities differ from selfassessed health as they also contain an expectational component that better predicts actual survival. This result supports the hypothesis that subjective beliefs on survival reflect private information, e.g., on the genetic background or on habits, and not biased beliefs. On the other hand, Elder (2013) shows a systematic bias in subjective survival forecasts due to individuals failing to capture fundamental properties of senescence, e.g., that annual death rates increase with age. Moreover, Smith, Taylor, and Sloan (2001) show that subjective survival probabilities do not incorporate all relevant information available to individuals in relation to their survival. Ludwig and Zimper (2013) propose a descriptive model with Bayesian learning where individuals tend to ignore relevant information about mortality as the prospect of death is approaching ("myside bias") leading to an overestimation of survival beliefs at older ages. Moreover, the model assumes an "initial" bias in beliefs that relates to ambiguity, because in the presence of ambiguity individuals tend to assess beliefs focusing on the worst case, which explains underestimation of beliefs at younger ages. In this modelling framework young individuals are generally pessimistic.

In this paper we propose a novel approach to investigate the role of behavioural biases in relation to mortality and longevity risks. We develop a simple model of sentiment similar to the one proposed by Barberis, Shleifer, and Vishny (1998) to study assets' re- 
turns. In our model pessimistic and optimistic expectations about the future health status co-exist and individuals update their beliefs about the prevailing sentiment based on their experienced health shocks. The model jointly predicts under-estimation of survival beliefs at younger ages and over-estimation at older ages assuming that individuals' optimism tend to persist over time, in line with psychological research. Differently from Ludwig and Zimper (2013), our model does not assume any age-dependent pattern in how individuals update information and does not impose any pessimistic prior. In our model younger individuals tend to over-react to health shocks (which are less frequent at their age), while older individuals tend to under-react to them, leading to the predicted pattern. The model also predicts that the magnitude of under- and over-estimation depends on individuals' priors with their pessimistic and optimistic expectations, and how persistent their sentiment is when an health shock realizes. The model shows that behavioural biases, in particular optimism, leads to biased survival beliefs and to the specific age-dependent pattern documented in the literature. We test the role of sentiment and the prediction of our model using the HRS data set and find significant support for the hypothesis that sentiment drives subjective beliefs.

The contribution of this paper is threefold. First, we develop a parsimonious theoretical model based on psychological evidence on optimism. The model produces the documented age-dependent pattern of subjective beliefs. Second, we apply a novel approach to determine actuarial probabilities. Differently from previous studies that take standard life tables as the actuarial counterpart to subjective beliefs, in this paper we construct actuarial probabilities taking into account existing information on individuals' health status and the specific time-frame they use to form their beliefs. This allows us to properly identify the role of psychological biases, while in previous studies the documented gap between subjective and actuarial probabilities could be due to under- or over-estimation of actuarial probabilities, which could explain the higher predictive power of subjective beliefs compared to their actuarial counterparts. Indeed, thanks to the way we compute objective survival beliefs, we clearly disentangle the objective component of subjective beliefs (e.g., diagnosed diseases) from the one driven by sentiment. We document that individuals tend to account for diagnosed diseases when they assess their health, but subjective beliefs tend to nevertheless reflect their optimistic sentiment, and under- or over-reaction to information. The third contribution lies in the economic implications of this study. Indeed, clarifying if subjective beliefs reflect private information or psychological biases is important, because it provides concrete directions on how to address observed mismatches between actual individuals' behaviour and the one predicted by standard economic theories. With 
the development of information economics, insurance has attracted the attention of economic and financial research (Cutler and Zeckhauser 2004). In particular, mortality and longevity risks, and the markets for life insurance and annuities, represent a suitable area to test standard economic theories. Related to this, vast empirical evidence has been collected about a mismatch between the practice of insurance and insurance theory (Cutler and Zeckhauser 2004, Kunreuther and Pauly 2004, and Liebman and Zeckhauser 2008).

There are some puzzling empirical observations if one analyses them with the lens of the classic theoretical foundation in economics. Our paper could represent a solid starting point to address these puzzles relying on biased subjective beliefs. For example, Yaari (1965) shows that expected utility maximizers with unknown death date should fully annuitize their savings, while empirical observations show that voluntary annuitization is rare. Davidoff, Brown, and Diamond (2005) prove that the so-called annuitization puzzle persists even under much weaker conditions than those considered by Yaari (1965) and advocate the use of behavioural biases to explain the puzzle; also see Brown et al. (2008) and Brown (2007b). Indeed, Benartzi, Previtero, and Thaler (2011) provide a behavioural perspective on the annuitization puzzle and emphasize the role of framing, default option, and institutional factors that could limit access to annuities. The implications of subjective survival beliefs for the explanation of patterns in the voluntary annuity market demand are also addressed by the economic and financial literature. From an empirical perspective, Inkmann, Lopes, and Michaelides (2011) find that subjective survival probabilities have differing effects on the annuitization decisions of non-stockholders and stockholders. Indeed, subjective probabilities are found to be statistically insignificant for nonstockholders, whereas a $10 \%$ increase in the baseline survival probability of stockholders significantly increases the annuity market participation probability by $0.75 \%$. From a theoretical perspective, $\mathrm{Wu}$, Stevens, and Thorp (2015) propose a model incorporating cohortand target- age-varying beliefs and show that deferred annuities are difficult products to market, because the perceived money's worth of these annuities turns out be very low at younger ages and increasing at advanced ages. O' Dea and Sturrock (2020) examine the role of mortality risk misperception in a framework where, while the annuity pricing depends on objectively measured survival probabilities, individuals' purchasing decisions are based on their own subjective survival probabilities. Whereas survival pessimism dominates, fairly priced annuities would be perceived as unfairly priced by individuals. Moreover, in the context of this study, survival pessimism is found to be quantitatively as important, or even more important, than the higher annuity prices determined by adverse selection in explaining rates of non-annuitization. The findings of the last two studies are 
not yet corroborated by any explanation for the age-dependent pattern of subjective beliefs. For instance, in Wu, Stevens, and Thorp (2015) a range of possible explanations is outlined, basing also on existing behavioural theories, e.g., the prospect theory of Kahneman and Tversky (1979).

Another puzzling observation is the insufficiency of life insurance among individuals in working age, especially among low-income households with children. Bernheim et al. (1999) and Bernheim et al. (2003) analyse data from the 1992 wave of the University of Michigan Health and Retirement Study (HRS) and find that around 30\% of wives and 10\% of husbands would have suffered a reduction of $20 \%$ or more of their living standard in case of their spouses dying (for $15 \%$ of women the reduction would have been of $40 \%$ or more). More recent evidence by Kim et al. (2020) and Hartley, Paulson, and Powers (2017) based on the Survey of Consumer Finances datasets suggests that ownership of life insurance significantly decreased from 1992 to 2016, and thus under-insurance at younger ages is likely to have worsen over the last two decades. A third puzzle we report here is the observation of excessive life insurance among the elderly (see Kim et al. 2020 and Hartley, Paulson, and Powers 2017). Brown (2007a) reports that in $199278 \%$ of coupled aged $70 \%$ or more owned life insurance. Despite ownership of life insurance decreased over the last two decades, in 2016 around 70\% of households aged 70\% or more possessed life insurance. Brown (2007a) considers some classical explanations for the high ownership of life insurance among the elderly population (e.g., the annuity offset model of Bernheim 1991), but concludes that none of them can be empirically confirmed. Instead, he refers to behavioural biases as a possible way to address the mismatch between theory and empirical observations. In relation to the ownership of life insurance, we also highlight here an additional interesting result. Empirical evidence provided by Kim et al. (2020, Figure 2) show that cash insurance and term insurance display different age-dependent patterns. Specifically, while ownership of cash insurance monotonically increases with age, ownership of term insurance reaches its maximum at around age 50-55 and decreases afterward. ${ }^{1}$ This pattern could be consistent with the documented dynamics of subjective beliefs, as under-estimation of survival at younger ages would imply that fairly priced term insurance products result more attractive, while over-estimation of survival at holder age would shift demand from term insurance to cash insurance, as the latter also contains a saving component and is more flexible in relation to the date of death.

Understanding if subjective beliefs reflect private information or psychological biases

\footnotetext{
${ }^{1}$ Differently from term insurance that pays off to survivors in case of death only, cash insurance contains a cash component that can be accessed by the policyholder when still alive to benefit for her living. Cash insurance premiums are higher than term insurance premiums.
} 
is important, because it allows to address with more awareness the current mismatch between the theory of insurance and the practice of insurance. The design of insurance products, e.g., annuities and life insurance, is affected by what drives subjective beliefs. Specifically, if individuals' expectations reflect private information, then insurance companies might want to further specialize their pricing structures, while if they reflect psychological biases or mistakes, then financial literacy or nudging might play an important role to improve decision-making.

The reminder of the paper is organized as follows. Section II presents the theoretical model and derives our main prediction. Section III reports our empirical analysis. Section IV concludes.

\section{Theoretical framework}

We present a model of sentiment similar to the one proposed by Barberis, Shleifer, and Vishny (1998). The model assumes that an individual switches between optimistic and pessimistic expectations on her health, also depending on her experienced health status $\eta$ up to age $x$.

The individual's health status evolves over time according to

$$
\eta_{x+1}=\eta_{x}+y_{x+1}
$$

where $y_{x+1} \in\{0, y\}, y<0, y_{0}=0$, and $\mathbb{P}\left[y_{x}=y\right]$ increases with $x$, i.e., older individuals face more frequently a deterioration of their health status compared to younger individuals. We assume that the health status can only deteriorate over time, i.e., $\eta_{x}$ decreases with age. This assumption captures an average pattern, or that an individual facing an health shock projects her health status into the future assuming the shock has long-term implications and affects her survival probability. In any case, our model's predictions do not depend on this assumption if the probability of negative shocks increases with the age.

The individual believes that $\left\{y_{x}\right\}_{x \geq 1}$ is driven by one of two models. Model 1 represents a pessimistic expectation on her future health and assumes a Markov structure for $\left\{y_{x}\right\}_{x \geq 1}$ with the transition probabilities as reported in Panel A of Table 1, where $\pi_{1 L}<0.5$ and $\pi_{1 H}>0.5$. By contrast, Model 2 represents an optimistic expectation on her future health and again assumes a Markov structure for $\left\{y_{x}\right\}_{t \geq 1}$ with the transition probabilities as reported in Panel B of Table 1, where $\pi_{2 L}<0.5$ and $\pi_{2 H}>0.5$. Under Model 1 the individual attributes a higher probability to a deterioration of her health status in the subsequent period, while under Model 2 the opposite applies. We also allow for different beliefs under 
Panel A: Model 1

\begin{tabular}{c|cc}
\hline & $y_{x+1}=0$ & $y_{x+1}=y$ \\
\hline$y_{x}=0$ & $\pi_{1 L}$ & $1-\pi_{1 L}$, \\
$y_{x}=y$ & $1-\pi_{1 H}$ & $\pi_{1 H}$, \\
\hline
\end{tabular}

Panel B: Model 2

\begin{tabular}{l|cc}
\hline & $y_{x+1}=0$ & $y_{x+1}=y$ \\
\hline$y_{x}=0$ & $\pi_{2 H}$ & $1-\pi_{2 H}$ \\
$y_{x}=y$ & $1-\pi_{2 L}$ & $\pi_{2 L}$ \\
\hline
\end{tabular}

Table 1: The table shows the transition probabilities under Model 1 (Panel A) and Model 2 (Panel B) for the Markow process $\left\{y_{x}\right\}_{x \geq 0}$ that describes health shocks at age $x$. We have $\pi_{1 L}<0.5, \pi_{1 H}>0.5$, $\pi_{2 L}<0.5$ and $\pi_{2 H}>0.5$.

both models depending on whether an health shock has just been observed or not. Finally, the individual is convinced to know how her sentiment $s_{x}$ changes over time. This is driven by a Markov process with the transition probabilities as reported in Table 2.

\begin{tabular}{c|cc}
\hline & $s_{x+1}=1$ & $s_{x+1}=2$ \\
\hline$s_{x}=1$ & $1-\lambda_{1}$ & $\lambda_{1}$ \\
$s_{x}=2$ & $\lambda_{2}$ & $1-\lambda_{2}$ \\
\hline
\end{tabular}

Table 2: The table shows the transition probabilities of the Markow process $\left\{s_{x}\right\}_{x \geq 0}$ that describes the sentiment at age $x$, where $s_{x}=1$ means Model 1 and $s_{x}=2$ means Model 2 .

We expect $\lambda_{2}$ to be small, reflecting the assumption that positive sentiment tends to be persistent over time. Empirical evidence shows that optimism and pessimism have age-dependent patterns (see Chopik, Kim, and Smith 2015). In our model we capture agedependent patterns as the consequence of health shocks, consistently with, e.g., the results of Chopik et al. (2020) showing that serious health shocks significantly lower optimism. We will also analyse all specifications of $\lambda_{1}$ and $\lambda_{2}$ to describe, for example, individuals who frequently change sentiment independently from specific life events. An extension of the model could also consider age-dependent values for $\lambda_{1}$ and $\lambda_{2}$ to better capture age-dependent effects of health shocks on sentiment, e.g., that middle-aged individuals' sentiment change more as a consequence of health shocks compared to older individuals, as found by Wurm, Tomasik, and Tesch-Römer (2009).

To complete the description of the model we finally assume that an individual survives time $T=x+h$ if and only if her health status at time $T$ is above a given threshold level $\eta^{\star}$, i.e., $\eta_{T} \geq \eta^{\star}$.

Individual's subjective belief about her survival depends on her expectation about her future health status and thus on her sentiment. Therefore, we start by computing the individual subjective probability that her sentiment will be according to Model 1 (pessimistic expectation) at age $x$, i.e.,

$$
\sigma_{x}\left(y_{x}, y_{x-1}, \sigma_{x-1}\right)=\mathbb{P}\left[s_{x}=1 \mid y_{x}, y_{x-1}, \sigma_{x-1}\right]
$$

The following lemma describes the dynamics of $\sigma_{x}$ : 


\section{Lemma 1.}

$$
\begin{aligned}
& \sigma_{x+1}\left(y_{x+1}, y_{x}, \sigma_{x}\right) \\
& =\frac{\mathbb{P}\left[y_{x+1} \mid s_{x+1}=1, y_{x}, \sigma_{x}\right]\left[\left(1-\lambda_{1}\right) \sigma_{x}+\lambda_{2}\left(1-\sigma_{x}\right)\right]}{\mathbb{P}\left[y_{x+1} \mid s_{x+1}=1, y_{x}, \sigma_{x}\right]\left[\left(1-\lambda_{1}\right) \sigma_{x}+\lambda_{2}\left(1-\sigma_{x}\right)\right]+\mathbb{P}\left[y_{x+1} \mid s_{x+1}=2, y_{x}, \sigma_{x}\right]\left[\lambda_{1} \sigma_{x}+\left(1-\lambda_{2}\right)\left(1-\sigma_{x}\right)\right]},
\end{aligned}
$$

where

$$
\mathbb{P}\left[y_{x+1} \mid s_{x+1}=1, y_{x}, \sigma_{x}\right]= \begin{cases}\pi_{1 L} & \text { if } y_{x+1}=y_{x}=0, \\ \pi_{1 H} & \text { if } y_{x+1}=y_{x}=y, \\ 1-\pi_{1 L} & \text { if } y_{x+1}=y \text { and } y_{x}=0, \\ 1-\pi_{1 H} & \text { if } y_{x+1}=0 \text { and } y_{x}=y .\end{cases}
$$

and

$$
\mathbb{P}\left[y_{x+1} \mid s_{x+1}=2, y_{x}, \sigma_{x}\right]= \begin{cases}\pi_{2 H} & \text { if } y_{x+1}=y_{x}=0, \\ \pi_{2 L} & \text { if } y_{x+1}=y_{x}=y, \\ 1-\pi_{2 H} & \text { if } y_{x+1}=y \text { and } y_{x}=0, \\ 1-\pi_{2 L} & \text { if } y_{x+1}=0 \text { and } y_{x}=y .\end{cases}
$$

Table 3 illustrates the dynamics of $\sigma_{x}\left(y_{x}, y_{x-1}, \sigma_{x-1}\right)$ for ages $x$ between 50 and 69 . We simulated health shocks $y_{x}$ according to the distribution $\mathbb{P}\left[y_{x}=0\right]=1-0.25 e^{0.03(x-102)}$ and iteratively computed $\sigma_{x}\left(y_{x}, y_{x-1}, \sigma_{x-1}\right)$ using the expression previously obtained and initiating the iteration with $\sigma_{0}=0$. We observe that a health shock strongly impacts $\sigma_{x}\left(y_{x}, y_{x-1}, \sigma_{x-1}\right)$. For example, at age 53 the individual suffers two subsequent health shocks and her probability $\sigma_{x}\left(y_{x}, y_{x-1}, \sigma_{x-1}\right)$ increases from 0.01 to 0.91 , i.e., the individual increased by 90 the probability assigned to her sentiment being pessimistic. Despite no health shock is observed in the following years after 54, we also see that it takes up to 4 years before $\sigma_{x}\left(y_{x}, y_{x-1}, \sigma_{x-1}\right)$ reaches the level before the shocks occurred.

\begin{tabular}{ccc|ccc}
\hline$x$ & $y_{x}$ & $\sigma_{x}$ & $x$ & $y_{x}$ & $\sigma_{x}$ \\
\hline 50 & 0.00 & 0.01 & 60 & 0.00 & 0.01 \\
51 & 0.00 & 0.01 & 61 & 0.00 & 0.01 \\
52 & 0.00 & 0.01 & 62 & 0.00 & 0.01 \\
53 & -0.10 & 0.53 & 63 & -0.10 & 0.53 \\
54 & -0.10 & 0.91 & 64 & 0.00 & 0.11 \\
55 & 0.00 & 0.35 & 65 & 0.00 & 0.02 \\
56 & 0.00 & 0.06 & 66 & 0.00 & 0.01 \\
57 & 0.00 & 0.02 & 67 & -0.10 & 0.53 \\
58 & 0.00 & 0.01 & 68 & 0.00 & 0.11 \\
59 & 0.00 & 0.01 & 69 & -0.10 & 0.68 \\
\hline
\end{tabular}

Table 3: The table shows the simulated dynamics of the subjective conditional probability $\sigma_{x}$ that the individual's sentiment is pessimistic. Age $x$ is between 50 and 69 years old. The parameters of the model are $y=-0.1, \lambda_{1}=\lambda_{2}=0.1, \pi_{1 L}=\pi_{2 L}=0.1$, and $\pi_{1 H}=\pi_{2 H}=0.9$. Moreover, $\mathbb{P}\left[y_{x}=0\right]=$ $1-0.25 e^{0.03(x-102)}$.

Figure 1 compares average probabilities $\sigma_{x}\left(y_{x}, y_{x-1}, q_{x-1}\right)$ and $\mathbb{P}\left[y_{x}=y\right]$. We observe 
that on average $\sigma_{x}\left(y_{x}, y_{x-1}, q_{x-1}\right)$ is above $\mathbb{P}\left[y_{x}=y\right]$ for younger individuals, while the opposite holds for older individuals. Because older individuals experience health shocks more frequently, they tend to react less strongly to new shocks, so their subjective probability assigned to the pessimistic model increases less than the probability of shocks' occurrence. This pattern is consistent with the empirical findings of Wurm, Tomasik, and Tesch-Römer (2009).

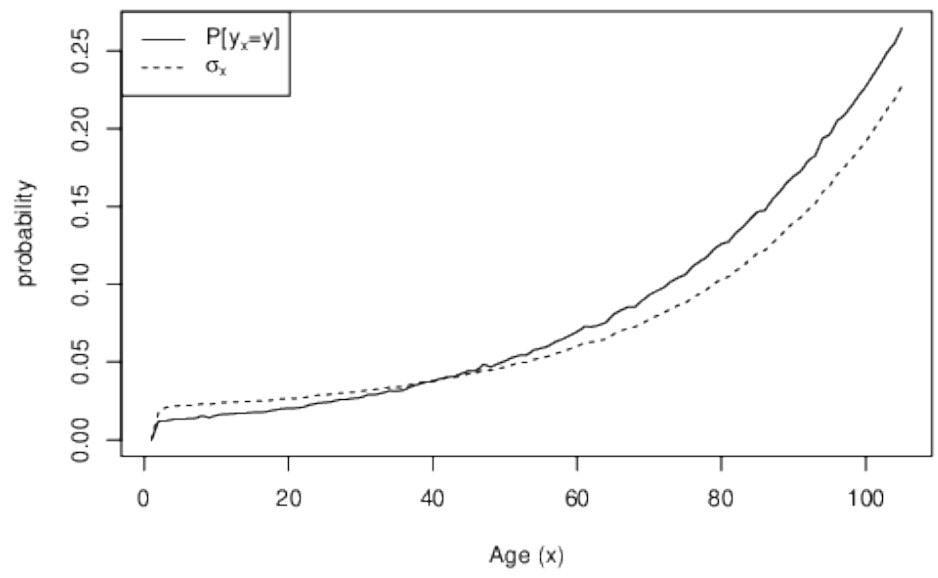

Figure 1: The figure shows the average subjective conditional probability for the pessimistic model $\sigma_{x}\left(y_{x}, y_{x-1}, q_{x-1}\right)$ (dotted line) and the average probability of an health shock $\mathbb{P}\left[y_{x}=0\right]=$ $1-0.25 e^{0.03(x-102)}$ (full line) at age $x$. We simulated 100,000 paths for $\left(y_{x}\right)_{x=0, \ldots, 105}$ based on $\mathbb{P}\left[y_{x}=y\right]$ and computed average values. We set $y=-0.1, \lambda_{1}=\lambda_{2}=0.1, \pi_{1 L}=\pi_{2 L}=0.1$, and $\pi_{1 H}=\pi_{2 H}=0.9$.

We now compute how subjective beliefs on sentiment affect the individuals' assessments of the survival probabilities. We consider an $x$-aged individual and her subjective probability to survive age $T=x+h$ given her current information about the health status $h_{x}$ and recent health shocks $y_{x-1}, y_{x}$ :

$$
{ }_{h} p_{x}^{s u b j}\left(\eta_{x}, y_{x}, y_{x-1}, \sigma_{x}\right)=\mathbb{P}\left[\eta_{x+h}>\eta^{\star} \mid \eta_{x}, y_{x}, y_{x-1}, \sigma_{x}\right]
$$

The following lemma provides the expression for the dynamics of ${ }_{h} p_{x}^{s u b j}$.

\section{Lemma 2.}

$$
\begin{aligned}
&{ }_{h} p_{x}^{s u b j}\left(\eta_{x}, y_{x}, y_{x-1}, \sigma_{x}\right)=\sum_{y_{x+1}}^{h-1} p_{x+1}^{s u b j}\left(\eta_{x}+y_{x+1}, y_{x+1}, y_{x}, \sigma_{x+1}\right) \\
& \times\left\{\mathbb{P}\left[y_{x+1} \mid s_{x+1}=1, y_{x}, \sigma_{x}\right]\left[\left(1-\lambda_{1}\right) \sigma_{x}+\lambda_{2}\left(1-\sigma_{x}\right)\right]\right. \\
&\left.\quad+\mathbb{P}\left[y_{x+1} \mid s_{x+1}=2, y_{x}, \sigma_{x}\right]\left[\lambda_{1} \sigma_{x}+\left(1-\lambda_{2}\right)\left(1-\sigma_{x}\right)\right]\right\}
\end{aligned}
$$


for $x=0,1, \ldots, x+h-1$ and

$$
{ }_{0} p_{x+h}^{s u b j}\left(\eta_{x+h}, y_{x+h}, y_{x+h-1}, \sigma_{x+h}\right)= \begin{cases}1 & \text { if } \eta_{x+h}>\eta^{\star} \\ 0 & \text { if } \eta_{x+h}<\eta^{\star}\end{cases}
$$

The following proposition considers a simplified case with two periods to provide insights on the conditions under which subjective beliefs over- and/or under-estimate survival probabilities.

Proposition 1. Let $h$ be such that $\eta_{x+h-1}>\eta^{\star}$ and $\eta_{x+h-1}+y<\eta^{\star}$, i.e., at time $x+h-1$ the individual is alive, but an health shock at time $x+h$ will be fatal. It follows that:

$$
\begin{aligned}
& { }_{1} p_{x+h-1}^{\text {subj }}\left(\eta_{x+h-1}, y_{x+h-1}, y_{x+h-2}, \sigma_{x+h-1}\right) \\
& \quad=\left\{\begin{array}{cl}
\left(1-\pi_{1 H}\right)+\left(\pi_{1 H}-\pi_{2 L}\right)\left[\lambda_{1} \sigma_{x+h-1}+\left(1-\lambda_{2}\right)\left(1-\sigma_{x+h-1}\right)\right] & \text { if } y_{x+h-1}=y, \\
\pi_{2 H}-\left(\pi_{2 H}-\pi_{1 L}\right)\left[\lambda_{2}\left(1-\sigma_{x+h-1}\right)+\left(1-\lambda_{1}\right) \sigma_{x+h-1}\right] & \text { if } y_{x+h-1}=0 .
\end{array}\right.
\end{aligned}
$$

Proposition 1 provides the main intuition for the implications of our model. Despite the proposition only addresses the two-period case, later we will also report simulation results showing that Proposition 1 holds more generally.

First of all, we consider younger individuals who are less likely to observe a health shock at time $x+h-1$. Therefore, we focus on the scenario $y_{x+h-1}=0$. In this case, the probability of survival ranges from $\pi_{2 H}$ to $\pi_{1 L}$ depending on $\lambda_{1}, \lambda_{2}$, and $\sigma_{x+h-1}$. Specifically, because $\sigma_{x+h-1}$ is likely to be small for younger individuals (as they generally experienced fewer health shocks), then a moderate-to-high $\lambda_{2}$ will shift subjective survival beliefs toward the pessimistic view, leading to an underestimation of survival beliefs compared to their actuarial counterpart which is rather high for younger individuals. Therefore, Proposition 1 predicts that underestimation of survival beliefs at younger ages is obtained when individuals' probability to change from an optimistic to a pessimistic sentiment is moderate-to-high. Panel A in Figure 2 reports the results of our simulations for the general case. We set $\pi_{1 L}=\pi_{2 L}=0.1$ and $\pi_{1 H}=\pi_{2 H}=0.9$, i.e., the probability of two subsequent shocks under Model 1 is high as well the probability of not having to subsequent shocks under Model 2. By contrast, the objective probability of having an health shock at age $x, \mathbb{P}\left[y_{x}=0\right]=1-0.25 e^{0.03(x-102)}$, is modelled with an exponential increase that generates realistic patterns for the objective survival probability. We see that the prediction of Proposition 1 generally holds in our theoretical setting. Indeed, the figure shows that the difference between subjective and actuarial beliefs at age 50 years old is negative for a wide range of parameters, i.e., our model predict an underestimation of survival at 50 years old. 
Specifically, we observe that values of $\lambda_{2}$ between 0.1 and 1 generate an underestimation of survival beliefs that ranges from $10 \%$ to $70 \%$.

We now consider older individuals who are more likely to observe an health shock at time $x+h-1$. Therefore, we now focus on the scenario $y_{x+h-1}=y$ in Proposition 1. In this case, the probability of survival ranges from $1-\pi_{1 H}$ to $1-\pi_{2 L}$ again depending on $\lambda_{1}, \lambda_{2}$, and $\sigma_{x+h-1}$. Specifically, because $\sigma_{x+h-1}$ is likely to be larger for older individuals compared to younger individuals (as they generally experienced more health shocks, see Figure 1), then a moderate-to-high $\lambda_{1}$ and a low-to-moderate $\lambda_{2}$ will shift subjective survival beliefs toward the optimistic view, leading to an overestimation of survival beliefs compared to their actuarial counterpart, which is rather low for older individuals. Therefore, Proposition 1 predicts that overestimation of survival beliefs at older ages is obtained when individuals' probabilities to change from a pessimistic to an optimistic sentiment is low-to-moderate. Panel B in Figure 2 reports the results of our simulations for the general case, again with parameters $\pi_{1 L}=\pi_{2 L}=0.1$ and $\pi_{1 H}=\pi_{2 H}=0.9$ and an exponential increase of the probability $\mathbb{P}\left[y_{x}=y\right]$ of the occurrence of the shock at age $x$. We see that the prediction of Proposition 1 generally holds in our model for older individuals as it was the case for younger individuals. Indeed, the figure shows that the difference between subjective and actuarial beliefs at age 75 years old is zero or positive, i.e., the model implies either accurate subjective beliefs or an overestimation of survival at 75 years old. Specifically, we observe that values of $\lambda_{1}$ between 0.6 and 1 together with values of $\lambda_{2}$ below 0.2 generate an overestimation of survival beliefs that ranges from $4 \%$ to $6 \%$. Our results are robust with respect to the choice of parameters $\pi_{i L}$ and $\pi_{i H}$ for $i=1,2$ as shown in Figure 4.

It follows that our model jointly predicts under-estimation of survival at younger ages and overestimation at older ages, when $\lambda_{1}$ is moderate-to-high and $\lambda_{2}$ is rather small as illustrated in Panel C of Figure 3. This case corresponds to when the optimistic sentiment tends to persist over time. However, because younger individuals are less likely to observe an health shock, when this realizes their reaction is excessive, while the opposite holds for older individuals, in line with empirical findings in Wurm, Tomasik, and Tesch-Römer (2009). We show in Figure 5 that this prediction is robust with respect to the choice of parameters $\pi_{i L}$ and $\pi_{i H}$ for $i=1,2$.

We emphasize that our theoretical prediction does not assume that older individuals are more optimistic than younger individuals. It simply states that individuals are generally optimistic ( $\lambda_{2}$ is small) and their sentiment is affected in the same way over the lifetime when an health shock realizes. However, while for younger individuals this leads 
to an overreaction, because shocks are less likely to seriously affect survival, the opposite holds for older individuals.

Panel A: Underestimation at 50 years old

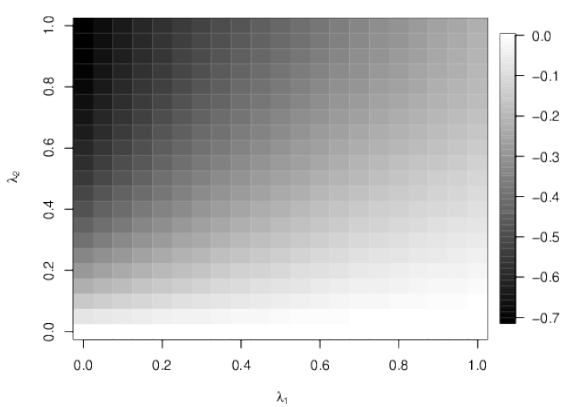

Panel B: Overestimation at 75 years old

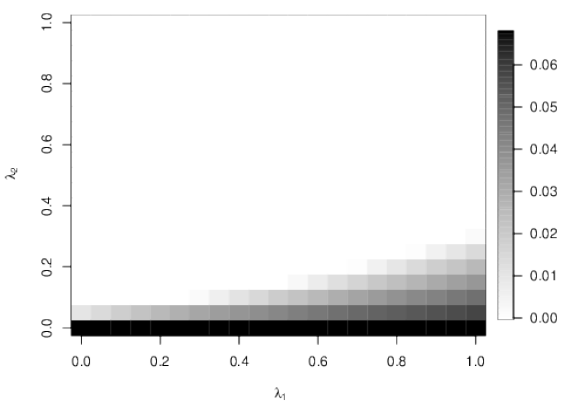

Figure 2: The figure shows the difference between the subjective and the objective probabilities of survival as a function of the parameters $\lambda_{1}$ and $\lambda_{2}$ at 0.05 steps. Otherwise we set $h=5$ years, $\pi_{1 L}=\pi_{2 L}=0.1$, and $\pi_{1 H}=\pi_{2 H}=0.9$. The objective probability of survival is computed assuming $\mathbb{P}\left[y_{x}=0\right]=1-0.25 e^{0.03(x-102)}$ to obtain a realistic pattern. At age $x=50$ years old (Panel A) the difference is always negative, that is the subjective probabilities underestimate survival. The degree of underestimation is illustrated with different grey colors, where black indicates a strong underestimation and white no underestimation. At age $x=75$ years old (Panel B) the difference is always positive, that is the subjective probabilities overestimate survival. Again the degree of overestimation is illustrated with different grey colors, where black indicates a strong overestimation and white no overestimation.

\section{Empirical analysis}

We now test the main predictions of our theoretical model. First of all, we verify if subjective beliefs imply under-estimation of survival at younger ages and over-overestimation at older ages. Previous studies already report evidence on this; see Hamermesh (1985), Perozek (2008), Zimper (2012), and Grevenbrock et al. (2021). However, we differentiate from these papers by how we compute the objective counterpart of subjective probabilities, as we derive health-dependent actuarial probabilities accounting for diagnosed diseases instead of only classifying individuals according to age and gender using standard life tables.

Second, we test whether the pattern is related to individuals' sentiment. Specifically, our model predicts that under-estimation and over-estimation occur when $\lambda_{2}$ is small, 
Panel A: Underestimation at $x=50$ years old only

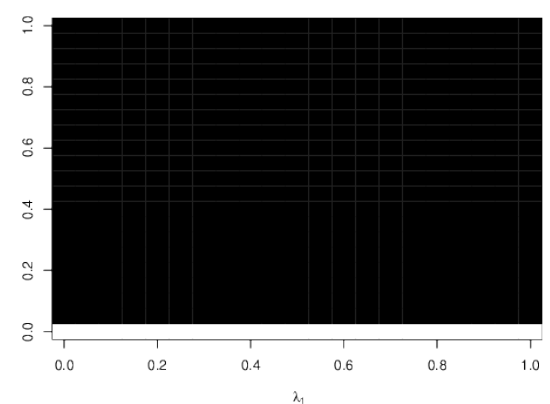

Panel B: Overestimation at $x=75$ years old only

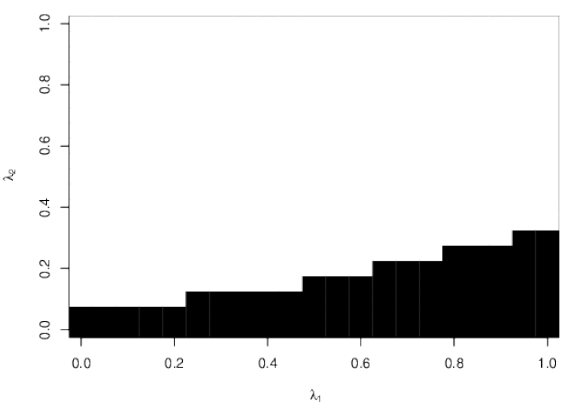

Panel C: Underestimation at 50 years old and overestimation at 75 years old

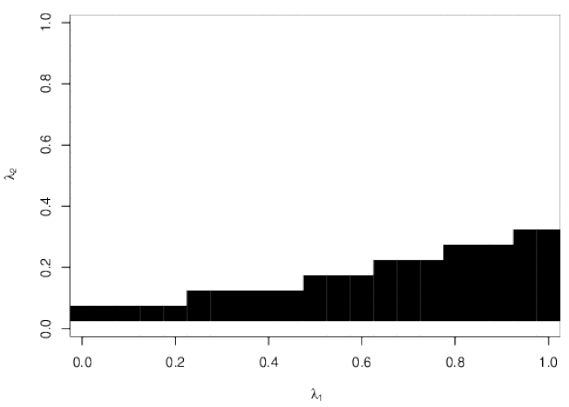

Figure 3: The figure shows the combinations of parameters $\lambda_{1}$ and $\lambda_{2}$ at 0.05 steps that imply underestimation of survival probabilities at $x=50$ years old (Panel A), overestimation at $x=75$ years old (Panel B) and both (Panel C). Otherwise we set $h=5$ years, $\pi_{1 L}=\pi_{2 L}=0.1$, and $\pi_{1 H}=\pi_{2 H}=0.9$. The objective probability of survival is computed assuming $\mathbb{P}\left[y_{x}=0\right]=1-0.25 e^{0.03(x-102)}$ to obtain a realistic pattern for actuarial probabilities of survival.

i.e., when individuals' optimism tend to be persistent, or when $\lambda_{1}$ is moderate-to-high and $\lambda_{2}$ is moderate, i.e., when individuals are more likely to transition from a pessimistic sentiment to an optimistic sentiment than the opposite. Our model also implies that the magnitude of under- and over-estimation is higher when individuals' sentiment is more extreme, i.e., under-estimation (over-estimation) decreases (increases) when the optimistic (pessimistic) prior is stronger, as described by the parameters $\pi_{k L}$ and $\pi_{k H}$ for $k=1,2$.

As mentioned above, the main issue we face in order to test our predictions is that we need a comparable objective (actuarial) counterpart to subjective probabilities. Because individuals are aware of existing diseases when formulating their subjective expectations, and, moreover, are deriving their beliefs using a specific time-frame depending on their 
Panel A: $\pi_{1 L}=\pi_{2 L}=0.01$ and $\pi_{1 H}=\pi_{2 H}=0.99$.
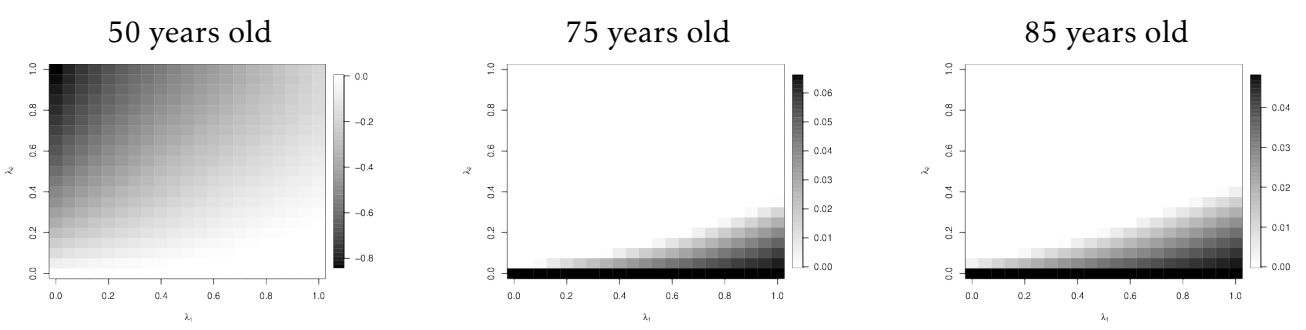

Panel B: $\pi_{1 L}=\pi_{2 L}=0.005$ and $\pi_{1 H}=\pi_{2 H}=0.995$.
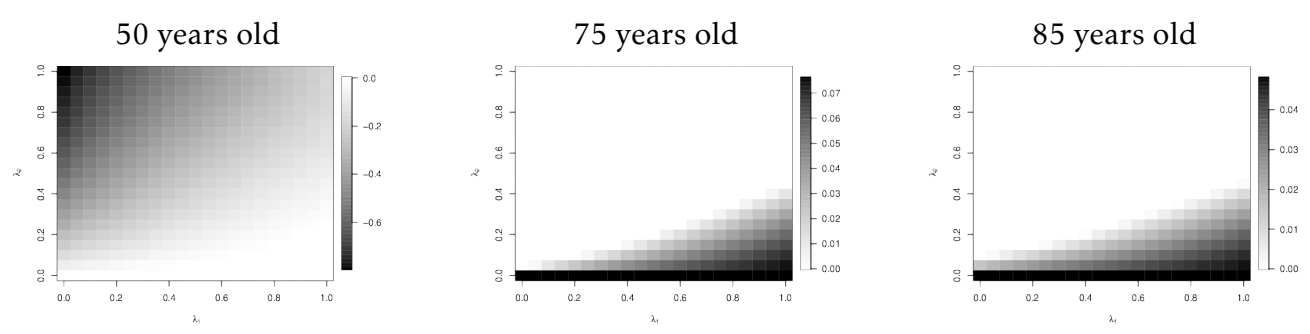

Figure 4: The figure shows the simulated difference between the subjective and the objective probabilities of survival at ages $x=50,75$, and 85 years old With $h=5$ years. The parameters $\lambda_{1}$ and $\lambda_{2}$ ranges from 0 to 1 at 0.05 steps. The objective probability of survival is computed assuming $\mathbb{P}\left[y_{x}=0\right]=1-0.25 e^{0.03(x-102)}$ to obtain a realistic pattern. In Panel A we set $\pi_{1 L}=\pi_{2 L}=0.01$ and $\pi_{1 H}=\pi_{2 H}=0.99$. In Panel B we set $\pi_{1 L}=\pi_{2 L}=0.005$ and $\pi_{1 H}=\pi_{2 H}=0.0995$. The degree of underestimation/overestimation of survival probabilities is illustrated with different grey colors, where black indicates a strong underestimation/overestimation and white no underestimation/overestimation.

age at the time they are interviewed, in Subsection B we construct health-dependent actuarial probabilities to reflect the same underlying information. In a second stage, in Subsections $\mathrm{C}$ and $\mathrm{D}$, we test our theoretical predictions on the gap between subjective and objective probabilities.

\section{A Data}

We use biennial data from the "University of Michigan Health and Retirement Study" (HRS), a longitudinal panel study supported by the National Institute on Aging (NIA U01AG009740) and the Social Security Administration surveying a representative sample of approximately 20,000 individuals in the United States of America ${ }^{2}$. In particular, we use data collected in the "waves" from 1996 to 2016, that is a properly long time horizon for our dynamic analysis. Each wave questionnaire consists of several thematic blocks or sections relating to a wide variety of domains. Each respondent is identified by an ID, fixed across waves and sections.

Within our study, we are mainly interested in the information that can be recovered from the following sections: cover sheet and demographics (e.g., data about the respon-

\footnotetext{
${ }^{2}$ More information about the Health and Retirement Study can be found here: https://hrs.isr.umich. edu/
} 
Panel A: $\pi_{1 L}=\pi_{2 L}=0.01$ and $\pi_{1 H}=\pi_{2 H}=0.99$.

Underestimation at 50 years old

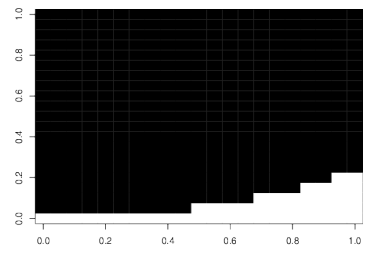

Overestimation at 75 years old

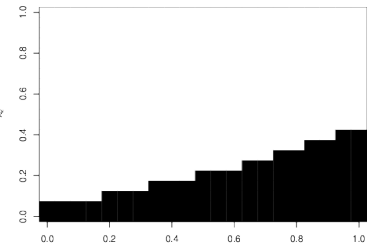

Underestimation and overestimation

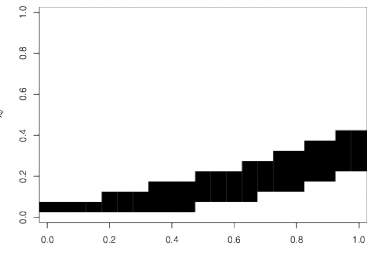

Panel B: $\pi_{1 L}=\pi_{2 L}=0.005$ and

$\pi_{1 H}=\pi_{2 H}=0.995$.

Underestimation at 50 years old

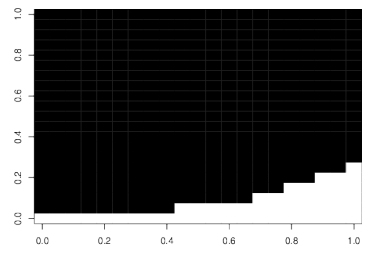

Overestimation at 75 years old

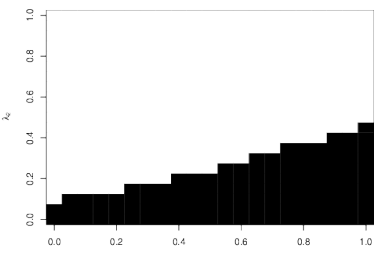

Underestimation and overestimation

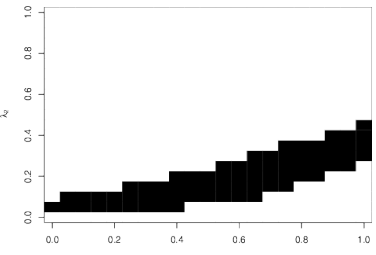

Figure 5: The figure shows the simulated difference between the subjective and the objective probabilities of survival at ages $x=50,75$, and 85 years old and $h=5$ years. The parameters $\lambda_{1}$ and $\lambda_{2}$ ranges from 0 to 1 at 0.05 steps. The objective probability of survival is computed assuming $\mathbb{P}\left[y_{x}=0\right]=1-0.25 e^{0.03(x-102)}$ to obtain a realistic pattern. In Panel A we set $\pi_{1 L}=\pi_{2 L}=0.01$ and $\pi_{1 H}=\pi_{2 H}=0.99$. In Panel B we set $\pi_{1 L}=\pi_{2 L}=0.005$ and $\pi_{1 H}=\pi_{2 H}=0.0995$. The degree of underestimation/overestimation of survival probabilities is illustrated with different grey colors, where black indicates a strong underestimation/overestimation and white no underestimation/overestimation.

dent's year of birth, gender, year of the interview, marital status, number of children and life satisfaction), health status (e.g., data concerning self-assessed health and disease conditions), expectations (e.g., survival expectations), and employment (e.g., data about the respondent's job status).

Subjective survival beliefs are values ranging from 0 to 100 answered to the question on the percent chance that the individual will survive a given target age set depending on the current age as indicated in Table 4 .

\section{A.1 Health status}

The concept of health is multidimensional. Indeed, one of the principles set out in the preamble to the World Health Organization (WHO) Constitution ${ }^{3}$ entered into force in April 1948, declares that "Health is a state of complete physical, mental and social well-

\footnotetext{
${ }^{3}$ The full text of the WHO Constitution can be found here: https://www.who.int/about/who-we-are/ constitution.
} 


\begin{tabular}{ccc}
\hline Age $(x)$ & \multicolumn{2}{c}{ Target age $(x+h)$} \\
& $1996-1998$ & $2000-2016$ \\
\hline $50 \leq x \leq 65$ & $75 \& 85$ & $75 \& 80$ \\
$66 \leq x \leq 69$ & 85 & 80 \\
$70 \leq x \leq 74$ & 85 & 85 \\
$75 \leq x \leq 79$ & 85 & 90 \\
$80 \leq x \leq 84$ & 85 & 95 \\
$85 \leq x \leq 90$ & & 100 \\
\hline
\end{tabular}

Table 4: The table reports target ages (columns 2 and 3) in dependence of current ages $x$ (column 1) as considered in the HRS for the question on survival expectations. For individuals aged between 55 and 65 two different target ages are considered, as shown in the second row of the table. Target ages have been adjusted in waves from 2000 to 2016 with respect to previous waves.

being and not merely the absence of disease or infirmity".

The HRS database includes exhaustive information about respondents' health status, considered in both its connotations: subjective and objective. Subjective health is measured by respondents' self-reported evaluations of their own health status through ratings ranging from excellent (1) to poor (5). Several studies in psychology and health psychology have investigated the validity of self-rated health as a predictor of future health outcomes (see Benyamini 2011 for an overview). One of the provided explanations endorsing the predictive ability of subjective health is that, being based on continuous monitoring of one's body and health, such a measure incorporates an amount of information that is largely unavailable to physicians and researchers. Self-assessed health and life satisfaction represent the two subjective well-being measures we consider in our study. In our empirical analysis we use life satisfaction as a proxy for individuals' sentiment. Sentiment and life satisfaction are generally different dimensions. However, the hypothesis that sentiment and life satisfaction are highly correlated is supported in a number of studies, e.g., Piper (2019) and Bailey et al. (2007). ${ }^{4}$

We assess respondents' objective health status by investigating the occurred diagnosis with one or more of the following disease conditions: high blood pressure, diabetes, cancer, emotional disorders and heart condition. We treat as "healthy" all those respondents who have never been diagnosed with any of these diseases. We choose to focus on the five mentioned health impairments since they remarkably increase individual frailty, thus involving a higher risk of mortality. According to the Global Health Observatory (GHO) data, made available by the World Health Organization in $2018^{5}$, heart-related conditions,

\footnotetext{
${ }^{4}$ The HRS study also provides direct information about sentiment (pessimism and optimism) in section "LB" (psycho-social). However, this section is optional for respondents and only about 10 of the them answer the corresponding questions. Therefore, in order to base our empirical analysis on a bigger data set, we preferred to use life satisfaction as a proxy of sentiment.

${ }^{5}$ See https://www.who.int/gho/mortality_burden_disease/causes_death/top_10/en/ for more information.
} 
such as heart disease and stroke, had been the leading causes of death worldwide in the last 15 years, causing 15.2 million deaths in 2016. In relation to the same year, some types of cancers (i.e., lung cancer) and diabetes mellitus were included among the top ten causes of death. It is also acknowledged in the literature that people suffering from mental disorders experience higher mortality rates than the general population (see Laursen, Nordentoft, and Mortensen 2013).

Strictly relating to our data, questions inquiring the presence of the five mentioned disease conditions are characterized by the highest response rates, this allowing us to subdivide the data sample into sufficiently large groups of individuals with homogeneous health characteristics, useful to obtain health-specific actuarial tables.

\section{B Actuarial probabilities: methodology}

Within our study, the subjective and the objective assessments of survival interface to each other. While individuals' survival beliefs express subjective expectations about the own residual lifetime, actuarial probabilities disclose information about how mortality is objectively expected to evolve, based on the forecasting outcomes of stochastic mortality models.

As explained by Mavros et al. (2017), the underlying assumption of stochastic mortality models is that a mixture of eventually independent deterministic and stochastic components, capturing age, period and, in some cases, cohort effects, explain human mortality dynamics. The broad range of models developed during the last two decades essentially differ from each other by the number and forms of the captured effects. Cairns et al. (2009) and Haberman and Renshaw (2011) provide quantitative comparative studies of the most influential and widely used mortality forecasting models, such as the models denoted by M1-M8. Model M1 represents the Lee-Carter method proposed by Lee and Carter (1992) and models from M5 to M8 are designed for shaping the mortality curve at pensioner ages. Such models assess systematic mortality evolution in that they are based on aggregate population level trends. More recent models (see Xu, Sherris, and Meyricke 2019) assess also the individual mortality heterogeneity arising from differing risk characteristics across individuals, such as socio-demographic characteristics and self-rated health.

For our investigation, we make use of the forecasts resulting from classical extrapolative stochastic mortality models disregarding individual heterogeneity, since these models represent the most deployed academic and industrial methods in modeling mortality rates. However, in order to obtain the most reliable benchmark to which subjective expectations are compared, we specialize the so predicted mortality rates to express the mortality expe- 
rience of individuals affected by a specific health status. In doing so, we implement analogous actuarial approaches to those traditionally used to assess the mortality of impaired assured individuals. Furthermore, for each health status under study we derive suitable mortality adjustment factors targeted to increase or decrease the average mortality rates characterizing the general population.

\section{B.1 Notation and actuarial foundation}

We now present our basic actuarial modeling framework. We denote by $T_{x}(t)$ the random residual lifetime for an individual aged $x$ at time $t$. Consequently, the conditional probability of surviving age $x+1$ given the information $\mathcal{I}_{t}$ at time $t$ is:

$$
p_{x}(t)=\mathbb{P}\left[T_{x}(t) \geq 1 \mid \mathcal{I}_{t}\right]
$$

Accordingly, we denote by $q_{x}(t)=1-p_{x}(t)$ the conditional probability given $\mathcal{I}_{t}$ that an individual aged $x$ at time $t$ will die before attaining age $x+1$. Because time $t$ could be located in the future, $p_{x}(t)$ and $q_{x}(t)$ are random variables.

From annual survival probabilities $p_{x}(t)$ we obtain all other relevant quantities. If $h$ is any positive integer, then the conditional probability given $\mathcal{I}_{t}$ that an individual aged $x$ at time $t$ survives age $x+h$, denoted by ${ }_{h} p_{x}(t)=\mathbb{P}\left[T_{x, t}>h \mid \mathcal{I}_{t}\right]$, corresponds to:

$$
{ }_{h} p_{x}(t)=\prod_{i=0}^{h-1} p_{x+i}(t+i) .
$$

Moreover, if $s>r$ are positive integers, then the conditional probability given $\mathcal{I}_{t}$ that an individual aged $x$ at time $t$ survives age $x+s$ given that she survives time $t+r$, denoted by ${ }_{r \mid s} p_{x}(t)=\mathbb{P}\left[T_{x, t} \geq s \mid T_{x, t} \geq r, \mathcal{I}_{t}\right]$, is given by:

$$
{ }_{r \mid s} p_{x}(t)=1-\left({ }_{r} p_{x}(t)-{ }_{s} p_{x}(t)\right)
$$

Finally, we define the force of mortality as

$$
\mu_{x}(t)=\lim _{h \searrow 0} \frac{h q_{x}(t)}{h}
$$

i.e., it corresponds to the instantaneous rate of mortality. Under certain assumptions (see Pitacco et al. 2009, Section 3.3.1) we have:

$$
q_{x}(t)=1-e^{-\mu_{x}(t)}
$$


Later in Subsection B. 3 we also consider the so-called crude death rate $m_{x}(t)$ for age $x$ in year $t$ to derive health-dependent mortality rates. The crude death rate is obtained by dividing the number $D_{x, t}$ of individuals aged $x$ dying in year $t$ by the so-called (central) exposure-to-risk $E_{x, t}$, i.e., the total time lived by individuals aged $x$ last birthday in year $t$ :

$$
m_{x}(t)=\frac{D_{x, t}}{E_{x, t}}
$$

$D_{x, t}$ and $E_{x, t}$ are observable and are used to fit our model. The realized values of these two variables are denoted by $d_{x, t}$ and $e_{x, t}$, respectively. Under certain assumptions, the crude death rate is the Maximum Likelihood estimator of the force of mortality, i.e., $\widehat{\mu}_{x}(t)=m_{x}(t)$ (see Pitacco 2010, Subsection 3.3.3).

\section{B.2 Mortality rates forecasting}

To obtain the actuarial counterpart of subjective survival beliefs, we fit and forecast the one-year probabilities of death $q_{x}(t)$. Applying Equation (4) and assuming that $\widehat{\mu}_{x}(t)=$ $m_{x}(t)$, we then compute the crude death rates $m_{x}(t)$, which we finally use to construct our health-dependent mortality rates.

We model $q_{x}(t)$ with the two-factor stochastic model known as CBD (or M5) model, proposed by Cairns, Blake, and Dowd (2006) for the development of the post-age-60 mortality curve through time. The choice of such a model is consistent with the age range (over 50) featuring the interviewed people in our HRS data sample. As the other Generalized Age-Period-Cohort stochastic mortality models, model M5 is characterized by a random and a systematic component connected by a link function. Following Haberman and Renshaw (2011) we assume that the random numbers of deaths $D_{x, t}$ are independent and binomially distributed conditionally on $E_{x, t}^{0}$ and $q_{x}(t)$ :

$$
D_{x, t} \sim \operatorname{Bin}\left(E_{x, t}^{0}, q_{x, t}\right)
$$

with $\mathbb{E}\left[\frac{D_{x, t}}{E_{x, t}^{0}}\right]=q_{x, t} . \quad E_{x, t}^{0}$ is the so-called initial exposure-to-risk that is approximated by adding half the matching reported number of deaths to the central exposure-to-risk $E_{x, t}$ (see Forfar, McCutcheon, and Wilkie 1988).

Concerning the systematic component, the following expression characterizes model M5 (see Cairns et al. 2009):

$$
\log \left(\frac{q_{x}(t)}{1-q_{x}(t)}\right)=k_{t}^{(1)}+k_{t}^{(2)}(x-\bar{x}),
$$


where $k_{t}^{(i)}, i=1,2$ are two time indices, and $\bar{x}$ is the mean age in the sample range. While the effect of the intercept or level term $k_{t}^{(1)}$ on the mortality dynamics is constant across ages, the slope $k_{t}^{(2)}$ exerts a larger effect at higher ages than at lower ages. As it appears in Equation (7), the mapping of the predictor structure to $q_{x}(t)$ involves a logit link function.

We perform the model calibration and prediction through the $R$ package StMoMo. ${ }^{6}$ For the period from 1933 to 2017, and for females and males separately, we collected the realized number of deaths $d_{x, t}$ and the realized central exposures-to-risk $e_{x, t}$ from the Human Mortality Database (2020). We finally computed the realized initial exposures to risk $e_{x, t}^{0}$. The two quantities $d_{x, t}$ and $e_{x, t}^{0}$ are the inputs for the estimation of the model.

Respondents in the HRS database formulate their expectations over a forecasting time horizon whose length depends on their current age $x$, as reported in Table 4. Moreover, we assume that individuals express their beliefs based on all information accrued during their life, i.e., for a number of years corresponding to their age at the interview time. We derive the actuarial counterpart of subjective probabilities using the same time frames. Specifically, we set the interview year as the observation year $t$ and an estimation window that spans the full individual's life, i.e., from $t-x$ to $t$. For example, for an individual aged 50 years old in 1996 who is asked to express her survival probability for the target age 75, we compute the actuarial counterpart based on the forecasted mortality rates $q_{51}(1997), q_{52}(1998), \ldots, q_{74}(2020)$. These probabilities are obtained according to Equation (7) estimated over the time interval from 1946 to 1996, i.e., the period the individual was alive. Since mortality data for the US population are available starting from 1933, when data do not fully cover an individual lifetime we base our estimation on data starting in 1933.

\section{B.3 Health-specific mortality rates}

Forecasted mortality rates as obtained in Section B.2 are the basic output of the CBD model that we adjust to reflect the average mortality experience of healthy and impaired individuals. Indeed, using HRS data we construct health-specific mortality tables and derive representative standardized death rates, evaluated against the death rates of the entire US population. As previously discussed, this step is very important to obtain a comparable objective counterpart to subjective probabilities. Our procedure is very close to the actuarial practice in the field of risk classification in life insurance, especially in relation to the underwriting of medical impairments (see, e.g., Hunter 1932 and Cummins et al. 1983).

\footnotetext{
${ }^{6}$ The packege has been developed by Villegas, Andres M., Pietro Millossovich, and Vladimir K. Kaishev. See https://cran.r-project.org/web/packages/StMoMo/StMoMo.pdf and https://cran. r-project.org/web/packages/StMoMo/vignettes/StMoMoVignette.pdf.
} 
We design and perform a period study with deaths and withdrawals (see Atkinson and McGarry 2016) between 1 January 2000 and 1 January 2017. Over this time period, we follow the cohorts of lives born between 1909 and 1949, namely all those individuals whose age at last birthday was between 50 and 90 at the start date of the study. These cohorts are selected from the national US population (the mortality data are collected from the HMD) and from the population of respondents included in the HRS-Wave 2000, unconditional on their health status. We call the two mentioned populations "HMD-national" and "HRS-total", respectively. We pool individuals from the HRS-total population according to available information about their health status at the start date of the study. We obtain 9 medical impairment classes, reflecting either only one specific disease among high blood pressure, diabetes, cancer, emotional disorders and heart condition or a combination of them (from 2 to 5 simultaneous impairments). We also build the class of healthy individuals, i.e., all respondents who had never been diagnosed with any of the mentioned diseases at the start year of the study.

In the HMD-national population only two events can affect an individual: survival or death. By contrast in the HRS-total population we have three events, because an individual can be either active in the HRS study and thus alive, or recorded as dead, or alive but in the meantime she has withdrawn from the study. We track the occurrence of such events collecting for each year and each of the 10 considered classes ( 9 medical impairment classes and the healthy class) the observed numbers of survivors $\left(l_{x, t}\right)$, deaths $\left(d_{x, t}\right)$, and withdrawals $\left(w_{x, t}\right)$. Data related to the intermediate calendar years when interviews are not performed can be easily derived from the available data. We compute the central exposure-to-risk $e_{x, t}$ according to Atkinson and McGarry (2016):

$$
e_{x, t}^{c}=\left(l_{x, t}-0.5 d_{x, t}-0.5 w_{x, t}\right) u_{x, t},
$$

where $u_{x, t}$ denotes the fraction of the year of age $x$ falling within year $t$. Finally, we derive crude death rates $m_{x, t}$ and one-year probabilities of death $q_{x, t}$ according to Equations (5) and (4), respectively.

The outcome of the described period study are mortality tables specific to each health class. From each table, we derive a unique average mortality rate, summarizing the mortality experienced by each class over the time period 2000-2016. In building this set of health-specific mortality rates, we have to account for the fact that health classes can differ by age composition. By way of an example, one health class can be characterized by a larger number of old people than the others. As a result, a higher mortality rate with 
respect to the other classes would also reflect the larger portion of the elderly in that class along with a general higher susceptibility to death for the people belonging to it. To make death rates suitable for between-classes comparisons, we have to adapt them for differences in the age-distribution of the various health classes, by computing what, in the actuarial practice, are known as age-standardized mortality rates. ${ }^{7}$ Such rates are indeed standardized with respect to a benchmark, that can be represented by the composition of a given national population in a given calendar year. Accordingly, an age-standardized mortality rate is the weighted average of age-specific mortality rates, where the weights are the proportions of people in the corresponding age groups of the standard population. We are guided by these principles when computing the age-standardized mortality rate representative of each health class. In particular, we consider that the population in each class is distributed in two age-groups, 50-64 and 65+, and we give the mortality rates for these two age-groups the same weights for all classes, namely the proportions of the exposed to risk in the corresponding age groups of the HMD-national population.

Relative mortality adjustment factors, denoted by $\gamma$, are computed by comparing the age-standardized mortality rate of each health class against the one relative to the HRStotal population. Adjustment factors, as shown in Table 5, are negative for the class of nonimpaired respondents and the following two classes of medical impairments: high blood pressure and emotional disorders. This implies that, based on the mortality data examined in our study, lives belonging to such classes represent "preferred" risks, since they exhibit a lower mortality profile with respect to the total population, whereas the risks assigned to the remaining medical impairment classes are "substandard" risks, experiencing a higher mortality than the total population.

The so obtained mortality adjustment factors quantify the differences in mortality rates between the aggregate HRS-total population and its sub-populations of healthy and impaired individuals. These relationships can be generalized to describe the discrepancies between the average mortality experienced by the HMD-national population and the specific mortality affecting healthy and impaired individuals. Therefore, the forecasted death rates based on the HMD-national population can be accordingly adjusted. Specifically, let $m_{x+k}(t+k)$ for $k=0,1, \ldots, h-1$ be the $k$-years-ahead forecasted death rates as obtained from the CBD model calibrated to the HMD mortality data as explained in Section B.2. For substandard risks $(\gamma>0)$ we apply the following multiplicative model (see Pitacco 2010,

\footnotetext{
${ }^{7}$ For more details on the concept of age-standardized mortality rate see, e.g., https://www. who.int/data/gho/indicator-metadata-registry/imr-details/78\#: : text=Definition3A, of20the20WH020standard20population. For computation methods, see Anderson and Rosenberg (1998).
} 


\begin{tabular}{lc}
\hline Class & $\gamma(\%)$ \\
\hline \multicolumn{2}{c}{ Preferred risks } \\
Healthy & -42 \\
High Blood Pressure & -31 \\
Emotional Disorders & -29 \\
\hline \multicolumn{2}{c}{ Substandard risks } \\
Cancer & 14 \\
Diabetes & 17 \\
Heart Condition & 23 \\
2 impairments & 23 \\
3 impairments & 92 \\
4 impairments & 157 \\
5 impairments & 707 \\
\hline
\end{tabular}

Table 5: The table reports the estimated adjustment factors $\gamma$ in percent for all considered 10 health-classes with respect to the average death rate of the HRS-total population. A negative (positive) adjustment means that death rates in the corresponding health class are smaller (larger).

page 90$)$ to obtain the health-adjusted mortality rates:

$$
m_{x+k}^{m}(t+k)=(1+\gamma) m_{x+k}(t+k)
$$

for $k=0, \ldots, h-1$. This model implies age-dependent adjustments $\gamma m_{x+k}(t+k)$ that increase with age since $m_{x+k}(t+k)$ increases with $k$. By contrast, for preferred risks $(\gamma<0)$ we use the following additive model:

$$
m_{x+k}^{a}(t+k)=m_{x+k}(t+k)+\gamma m_{x}(t)
$$

for $k=0, \ldots, h-1$. Indeed, for preferred risks the multiplicative structure is not suitable, because a downward adjustment that increases with age is not realist. By contrast, the additive model involves a constant adjustment $m_{x}(t)$, independent from the age.

\section{Gap between subjective and actuarial probabilities}

Using the actuarial probabilities obtained as described in the previous subsection we compute and analyse the gap between subjective survival probabilities and their actuarial counterparts. For each $x$-aged individual interviewed in year $t$, we compare the stated subjective probability of surviving $h$ years, ${ }_{h} p_{x}^{s u b j}(t)$, with its actuarial counterpart, ${ }_{h} p_{x}^{a c t}(t)$. We tested our prediction that younger individual underestimate survival probabilities $\left.{ }_{h} p_{x}^{s u b j}(t){ }_{h} p_{x}^{a c t}(t)<0\right)$, while older individual overestimate survival probabilities $\left({ }_{h} p_{x}^{s u b j}(t)-{ }_{h}\right.$ $\left.p_{x}^{a c t}(t)>0\right)$. The results are reported in Table 6 for ages in our sample for which subjective probabilities have been formulated over a forecasting horizon of $h=11$ years, i.e., 
$x=64,69,74,79,84$ and $89 .{ }^{8}$ Focusing on a fixed forecasting period of $h=11$ years for all ages allows to examine how individuals' attitudes towards longevity change with age and thus to identify age-dependent patterns.

\begin{tabular}{lcccccc}
\hline Gender & Age & $\begin{array}{c}\text { Subjective } \\
(x)\end{array}$ & $\begin{array}{c}\text { Actuarial } \\
\left({ }_{h} p_{x}^{\text {subj }}\right)\end{array}$ & $\begin{array}{c}\text { Difference } \\
\left({ }_{h} p_{x}^{a c t}\right)\end{array}$ & $\begin{array}{c}\text { p-value } \\
\left({ }_{h} p_{x}^{s u b j}-\overline{{ }_{h} p_{x}^{a c t}}\right)\end{array}$ & N.obs. \\
\hline \multirow{2}{*}{ Female } & 64 & 67.76 & 81.13 & -13.37 & 0.00 & 433 \\
& 69 & 61.20 & 72.41 & -11.21 & 0.00 & 362 \\
& 74 & 54.88 & 55.85 & -0.97 & 0.63 & 266 \\
& 79 & 43.86 & 37.07 & 6.79 & 0.00 & 242 \\
& 84 & 35.22 & 22.87 & 12.35 & 0.00 & 122 \\
& 89 & 32.59 & 10.26 & 22.33 & 0.00 & 51 \\
\hline Male & 64 & 64.40 & 71.53 & -7.13 & 0.00 & 392 \\
& 69 & 59.38 & 58.45 & 0.93 & 0.61 & 309 \\
& 74 & 57.69 & 43.66 & 14.03 & 0.00 & 215 \\
& 79 & 42.60 & 27.16 & 15.44 & 0.00 & 168 \\
& 84 & 43.66 & 16.98 & 26.68 & 0.00 & 82 \\
& 89 & 33.39 & 6.53 & 26.86 & 0.00 & 31 \\
\hline
\end{tabular}

Table 6: The table reports average subjective $\overline{\left({ }_{h} p_{x}^{s u b j}\right.}$ ) and actuarial $\overline{h_{h}^{a c t}}$ survival probabilities, and their average differences $\overline{{ }_{h} p_{x}^{\text {subj }}}-\overline{{ }_{h} p_{x}^{a c t}}$ for selected ages $(x)$, and target ages $x+11$, i.e., the forecasting horizon is $h=11$ years for all ages. All reported probabilities are expresses with percentages. We tested the hypothesis that differences are zero against the alternative they are different from zero using a Welch's t-test. The $p$-values are reported in Column 6.

Table 6 reveals two main empirical results. First, younger (older) individuals underestimate (overestimate) survival probabilities with respect to their actuarial counterparts, consistently with the prediction of our model when individuals are generally optimistic. Second, subjective probabilities decrease more slowly as a function of age than their actuarial counterparts. This latter result is also in line with our model of sentiment when individuals are generally optimistic. These observed patterns are similar for female and male participants, despite the turning point for male respondents is lower (at around 70 years old) compared to female respondents (at around 74 years old). This latter result further support our prediction based on sentiment (i.e., the role of priors as described by $\pi_{i L}$ and $\pi_{i H}$ for $\left.i=1,2\right)$, as the empirical and experimental evidence shows that males are generally more optimistic than females; see Bjuggren and Elert (2019). Table 6 only considers ages with a forecasting horizon of 11 years. However, underestimation at younger ages and overestimation at older ages is consistent across all ages and their corresponding target ages. Indeed, the average underestimation for females aged between 50 and 74 is $-10.25 \%$ (st.dev. is $5.49 \%$ ) and the average overestimation for females aged 75 or more is $14.48 \%$ (st.dev. is $6.80 \%$ ). Similarly, the average underestimation for males aged between 50 and 69 is $-6.21 \%$ (st.dev. is 3.34\%) and the average overestimation for males aged 70 or

\footnotetext{
${ }^{8}$ In order to be able to fix the time horizon to 11 years for all individuals, we restrict our analysis in this subsection to waves from 2000 to 2016.
} 
more is $19.12 \%$ (st.dev. is $5.01 \%$ ).

Our findings partially coincide with those reported by Hamermesh (1985), Hurd and McGarry (2002), and Perozek (2008). However, we emphasize that in this paper we apply a different methodology to obtain actuarial probabilities. Indeed, we compute actuarial probabilities accounting for existing, reported diseases and individuals' time-frames, while previous studies do not, and use standard life tables. Therefore, the gaps we report are generally higher, specifically for older individuals, reaching almost $27 \%$ for 89 -aged males forecasting their probability to reach target age 100. Moreover, Perozek (2008) focused on wave 1992 and only on younger male and female individuals aged between 52 and 57, respectively, for which overestimation is found for male participants and underestimation for female participants. This result also supports our explanation based on sentiment given the documented gender gap in optimism.

Perozek (2008) and Hurd and McGarry (2002) argue that the documented gap in survival probabilities reflect the fact that individuals can exploit private information on their health to assess survival expectations. Indeed, respondents in the HRS study have access to information concerning their own genetic background and the environmental and behavioural risk factors they are exposed to, such as exercise, diet, and smoking habits. Perozek (2008) and Hurd and McGarry (2002) show that subjective probabilities differ from self-assessed health. Therefore, the former seems to also contain an expectational component, e.g., related to habits or to the death of a parent, and better predicts actual survival compared to self-assessed health.

We do not contrast the predictive power of subjective probabilities, but argue that average gaps between subjective and actuarial probabilities ranging from $-15.33 \%$ to $27.54 \%$, can hardly be explained with individuals exploiting private information only. Specifically, because we adjusted actuarial probabilities to account for diagnosed diseases, the composition of the HRS-population analysed, and the time frames over which individuals form their beliefs, the documented average under- and overestimation of survival beliefs would imply that private information leads to much lower average expectations for younger individuals and to much larger expectations for older individuals, suggesting that private information is generally negative for younger individuals and generally positive for older individuals. Related to this, Smith, Taylor, and Sloan (2001) show that subjective survival probabilities do not incorporate all relevant information available to individuals in relation to their survival.

Therefore, our hypothesis is that under-estimation at younger ages and over-estimation of older ages is mainly due to sentiment. Indeed, the model of sentiment we propose in 
the theoretical part of the paper predicts under- and over-estimation of survival probabilities assuming that individuals are generally optimistic. However, at younger ages, when health shocks are less frequent and less relevant for survival, individuals tend to overreact to them, which leads to under-estimation of survival. Oppositely, at older ages, despite health shocks are more frequent, the positive sentiment still prevails and leads to an overestimation of survival. Our theoretical prediction does not assume that older individuals are more optimistic than younger individuals. It simply states that individuals are generally optimistic and their sentiment is affected in the same way when an health shock realizes during their lifetime. However, while for younger individuals this leads to an overreaction because shocks are less likely to seriously affect survival (as captured by actuarial probabilities), the opposite holds for older individuals.

This feature of our model of sentiment allows us to clearly distinguish our contribution from previous papers also suggesting a behavioural explanation to the observed gap in survival probabilities. Specifically, Ludwig and Zimper (2013) developed a model of Bayesian learning encompassing rational Bayesian learning and accounting for psychological biases in the interpretation of new information. Such biases relate to the attitude toward likelihood insensitivity and optimism, both becoming stronger with increasing age. Their assumption is that "psychological biases in subjective survival expectations may vary with age because cognitive encounters with death are stronger when the risk becomes more relevant." By contrast, our model of sentiment does not assume that psychological biases vary with age. In the next subsection we provide empirical evidence in favour of this assumption.

\section{Subjective probabilities and sentiment}

\section{D.1 Life satisfaction as a proxy of sentiment}

To test our hypothesis on the role of sentiment in deriving subjective beliefs, we need to specify a variable that captures individuals' sentiment. The HRS study provides direct information about sentiment (pessimism and optimism) in section "LB" (psycho-social). However, this section is optional for respondents and only about 10 of the them answer the corresponding questions. Therefore, in order to base our empirical analysis on a bigger data set, we verify if reported life satisfaction can be used as a proxy of sentiment in combination with gender. Indeed, we also rely on empirical evidence showing that men tend to be more optimistic than women, so gender already partially accounts for a possible differential in sentiment; see Jacobsen et al. (2014) and Bjuggren and Elert (2019). However, to also capture the dynamics of subjective beliefs, gender is not sufficient as a 
proxy of sentiment, and thus we add a variable that describes inter-gender differences in sentiment.

There is empirical evidence showing that optimism, being the stable tendency to expect good future outcomes, is a predictor of individuals' attitude toward life, as measured by life satisfaction indexes (see Piper 2019 and Bailey et al. 2007). Cummins and Nistico (2002, Page 37) suggest that life satisfaction "is not simply under the influence of experience but is managed, by some form of psychological process, such that a positive view of life predominates". This hypothesis aims at explaining the high and stable level of life satisfaction typically reported. They review a number of studies empirically supporting their hypothesis, highlighting the high correlation between measured life satisfaction and optimism. Moreover, optimistic individuals are more able to reappraise negative events (see Bryant and Cvengros 2004 and Grover 2020), leading to a stable level of life satisfaction.

In the HRS study data about respondents' satisfaction with their life-as-a-whole are included in the "Demographics" module of waves from 2008 to 2016. Self-reported evaluations of life satisfaction are expressed through ratings ranging from 1 (completely satisfied) to 5 (not at all satisfied). We validate this variable by using regression analysis to prove that: (i) the subjective measure of the quality of life, as expressed by respondents in the HRS study, is a reliable measure of satisfaction with life; (ii) life satisfaction reflects individuals' optimistic attitude.

Life satisfaction judgements are based on personality dispositions and circumstance factors such as satisfaction with specific life domains, e.g., marital status, job status, health (see Pavot and Diener 2007). To verify (i) we estimate, through an OLS regression, the relationship between reported life satisfaction and a set of individual observable characteristics describing some of the life domains previously mentioned. We also include explanatory variables on individuals' subjective assessment of their health status, namely self-assessed health as described in Subsection A.1. The results are reported in Table 7.

The average life satisfaction in our reference category (males, aged between 50 and 64, married, and currently employed) is 1.66 , i.e., between completely satisfied and very satisfied. Moreover, because the average variation in other categories is lower than 0.3 , we conclude that average life satisfaction is high in all categories. Indeed, 65\% of the respondents in our sample declared themselves between completely satisfied and very satisfied about their overall life. Moreover, older individuals (age above 64) are generally more satisfied than younger individuals. The age-effect in our sample is in line with a large body of empirical literature in social sciences, psychology, and medicine documenting that wellbeing reaches its minimum around 50 years old, resulting in a consistent $U$-shape pattern 


\begin{tabular}{lccc}
\hline Variable & Estimate & St.Error & p-value \\
\hline Intercept & 1.66 & 0.02 & 0.00 \\
Female & -0.01 & 0.01 & 0.19 \\
Age group $>$ 64 years & -0.14 & 0.01 & 0.00 \\
N. diseases & 0.01 & 0.01 & 0.02 \\
Self-assessed health & 0.23 & 0.01 & 0.00 \\
Never Married/Annulled & 0.24 & 0.02 & 0.00 \\
Separated/Divorced & 0.30 & 0.01 & 0.00 \\
Widowed & 0.21 & 0.02 & 0.00 \\
Unemployed and looking for a job & 0.29 & 0.03 & 0.00 \\
Temporarily laid off, on sick or other leave & 0.17 & 0.05 & 0.00 \\
Disabled & 0.04 & 0.02 & 0.02 \\
Retired & -0.14 & 0.02 & 0.00 \\
Homemaker & -0.10 & 0.02 & 0.00 \\
Other job status & 0.07 & 0.06 & 0.29 \\
N. children & -0.01 & 0.00 & 0.03 \\
\hline Observations & 26,147 & & \\
\hline
\end{tabular}

Table 7: The table reports the OLS regression results for life satisfaction. Individual life satisfaction rating (ranging from 1 - completely satisfied - to 5 - not at all satisfied) is the dependent variable. Gender, age, marital status, and job status are categorical variables. The respective reference categories are males, aged between 50 and 64, married, and currently employed.

in life satisfaction across several countries (see, for example, Blanchflower 2020). In relation to this, we also observe that retired individuals are generally more satisfied compared to the reference category of employed individuals. By contrast, we do not identify any significant difference in life satisfaction between males and females; see Joshanloo and Jovanović (2020) and the extensive literature cited therein showing that there is mixed evidence in relation to a gender gap in life satisfaction, despite the difference in optimisms documented in the literature.

Our results also show that married individuals and employed individuals are more satisfied than unemployed and unmarried individuals, respectively. The number of children also increases life satisfaction, despite the effect is small. Finally, the health status (as self-assessed by the individuals) affects life satisfaction, despite only marginally as a one-point-increase (again in a 1-to-5 scale, where 1 means excellent and 5 means poor) of self-assessed health leads to an average increase of 0.23 points of the rating for life satisfaction. Later we will report additional evidence showing that life satisfaction is only marginally affected by self-assessed health, despite the latter tends to properly account for diagnosed diseases. Overall we observe that on average reported life satisfaction properly accounts for individuals' satisfaction in relation to important domains of life.

We now test if the reported life satisfaction is a proper measure of sentiment, i.e., correlates with optimistic expectations outside the specific domain of one's life. Within the "Expectations" module of the HRS study the interviewed people are faced with several questions asking them to quantify the chance that a given event will happen over a given 
time horizon. Among the questions included in all waves from 2010 to 2016, we selected the question asking about the chance that, within one year "mutual fund shares invested in blue chip stocks (like those in the Dow Jones Industrial Average) will have fallen in value by more than 20 percent compared to what they are worth today?" Objective expectations about financial markets shouldn't depend on individuals' characteristics. By contrast, subjective probabilities could reflect individuals' sentiment at the time the interview is performed. Specifically, based on historical data, a decrease in value by more than $20 \%$ within a year is a not-so-likely event for a well-diversified portfolio. ${ }^{9}$ However, individuals' expectations, also depending on the overall sentiment, are affected by daily changes on the stock market, which are then projected to assess the likelihood of strong market corrections within a year (see Benartzi and Thaler 1995). Therefore, to test if reported life satisfaction captures sentiment, we run a OLS regression on individuals' expectations about the performance of the stock market with respect to their characteristics, including life satisfaction. Table 8 reports the results.

We observe that individuals in our sample are generally pessimistic about the performance of the financial market. Indeed, we estimated an average probability of $36.77 \%$ for a loss of $20 \%$ or more within one year in our reference category (males, aged between 50 and 64, assessing their health status as excellent, completely satisfied about their life, married and currently employed). This result could be due to misjudgements (i.e., the risk perception is affected by daily returns) or to the fact that individuals were experiencing high losses during the financial crisis (the S\& P500 index lost 56.8\% from October 2007 to March 2009) and their expectations are affected by past performance, as suggested by Greenwood and Shleifer (2014). ${ }^{10}$

We also observe that older (aged 65 and more), married, and retired individuals assign a significantly lower $(8.58 \%$ lower $)$ average probability to a considerable market correction compared to our reference categories. Moreover, we also identify a significant gender effect, with female respondents being more pessimistic about the performance of welldiversified portfolios (see Jacobsen et al. 2014). Because we didn't identify a gender gap in life satisfaction, we interpret the gender gap as the effect of the lower optimism among females compared to males. Therefore, gender partially captures sentiment. Finally, we also find a significant effect of life satisfaction on individuals' expectations concerning financial markets, supporting the hypothesis that reported life satisfaction is driven by in-

\footnotetext{
${ }^{9}$ Based on Robert Shiller monthly data for the U.S. Stock Market (http: / / www . econ. yale.edu/ shiller/ data.htm), starting from 1871 only around $6.5 \%$ of returns over one year were below $-20 \%$, and only 12 dropdowns were larger than $20 \%$. If we limit the analysis to post-1950 market returns, we have $3.7 \%$ of returns over one year below $-20 \%$.

${ }^{10}$ The results in Table 8 are derived from all waves of the HRS from 2010 to 2016.
} 


\begin{tabular}{lrcc}
\hline Variable & Estimate & St.Error & p-value \\
\hline Intercept & 36.77 & 1.02 & 0.00 \\
Female & 3.48 & 0.42 & 0.00 \\
Age group > 64 years & -5.45 & 0.58 & 0.00 \\
N. children & 0.21 & 0.10 & 0.05 \\
N. diseases & 0.06 & 0.21 & 0.77 \\
\hline Self-assessed health & & & \\
Very good & -0.68 & 0.71 & 0.33 \\
Good & 1.22 & 0.73 & 0.09 \\
Fair & 1.48 & 0.83 & 0.07 \\
Poor & 0.90 & 1.12 & 0.42 \\
\hline Marital status & & & \\
Never Married/Annulled & -1.74 & 0.83 & 0.04 \\
Separated/Divorced & 0.77 & 0.55 & 0.16 \\
Widowed & -1.21 & 0.66 & 0.06 \\
\hline Job status & & & \\
Unemployed and looking for a job & 0.67 & 1.00 & 0.50 \\
Disabled/Temporarily laid off, on sick or other leave & 0.77 & 0.77 & 0.32 \\
Retired & -1.39 & 0.64 & 0.03 \\
Homemaker/Other job status & 0.50 & 0.91 & 0.59 \\
\hline Life satisfaction & & & \\
Very satisfied & 0.20 & 0.53 & 0.70 \\
Somewhat satisfied & 1.76 & 0.75 & 0.02 \\
Not very/not at all satisfied & 2.62 & 0.68 & 0.00 \\
\hline Observations & 15,090 & & \\
\hline
\end{tabular}

Table 8: The table reports the OLS regression results for stock market expectations. Stock market expectations is the dependent variable. Stock market expectations are expressed as the probability (in percentage) that mutual fund shares invested in blue-chip stocks like those in the Dow Jones Industrial Average will fall by more than $20 \%$ within one year. Gender, age, self-assessed health, life satisfaction, marital status, and job status are categorical variables. The respective reference categories are males, aged between 50 and 64, self-assessing their health as excellent, extremely satisfied about their life, married, and currently employed.

dividuals' general attitude toward life, i.e., their positive cognitive biases, as theorized by the homeostasis model (see Cummins 1998).

We tested additional questions in the "Expectations" module of HRS. We found that life satisfaction and gender generally predict lower optimism. This supports the use of life satisfaction as an additional proxy for sentiment on the top of gender.

\section{D.2 Determinants of subjective probability}

The previous section empirically tested the existence of a gap between subjective and actuarial survival probabilities. We now apply regression analysis to identify which of the documented individuals' characteristics better explains subjective survival probabilities. To this aim, we use collected data of 9,724 individuals participating in one of the HRS waves from 2008 to 2016 . Therefore, the analysis in this subsection is static, while in the next subsection we address how subjective probabilities dynamically change over time.

Consistently with how we forecasted actuarial death rates $q_{x}(t)$ (see Subsection B.2), we transform subjective survival probabilities ${ }_{h} p_{x}^{s u b j}(t)$ using a logit function before running 
a generalized linear regression model. To deal with 0 and 1 values (which are present in our sample as individuals tend to round numerical responses to the nearest 5-10, see, e.g., Manski 2004 and Manski and Molinari 2010) we follow Blass, Lach, and Manski (2010) and make an ad-hoc transformation of reported zeroes and ones to 0.001 and 0.999 , respectively. ${ }^{11}$

Table 9 reports the estimated parameters of our regression model for subjective probabilities. We observe that female respondents tend to express higher survival probabilities, consistently with actuarial estimates, despite the gender difference is $50 \%$ lower with subjective expectations compared to their objective counterparts. This result suggests that female participants generally account for their higher probability of survival compare to male participants, but much less than they should, so behaving more pessimistically. Our regressions results also show that older individuals (aged 65 or more) reports lower subjective survival probabilities than younger individuals. However, the difference is much smaller than that of the actuarial counterpart, in line with the results of the previous subsection showing that subjective probabilities with comparable forecasting horizons decrease more slowly as a function of age compared to the actuarial estimates.

Differently from actuarial estimates, individuals do not seem to consider the number of existing diseases to form their beliefs about survival. By contrast, their own assessment of the health status plays a significant role, as subjective probabilities are lower for individuals classifying their health status as inferior. Later we provide evidence that self-assessed health partially accounts for occurred health shocks. Therefore, individuals' subjective assessments of survival also include an objective component. However, we also identify the impact of sentiment as captured by life satisfaction. Specifically, individuals with lower levels of life satisfaction increasingly reduce subjective expectations. This effect is statistically significant, despite we control for all variables predicting life satisfaction as reported in Table 7.

\section{D.3 Updating of subjective probabilities and sentiment}

In this subsection we take a dynamic perspective and analyse how individuals update subjective probabilities in the presence of new information. The goal is now to identify the role of sentiment when individuals are confronted with an health shock or remain healthy.

Our analysis in this subsection is based on all waves of the HRS study between 1996

\footnotetext{
${ }^{11}$ We checked robustness of our estimates by performing a linear median regression model, which is not sensitive to our rounding procedure. The results confirm the findings with the generalized linear regression model.
} 


\begin{tabular}{lccc}
\hline Variable & Estimate & St.Error & p-value \\
\hline Intercept & 0.91 & 0.10 & 0.00 \\
Female & 0.22 & 0.04 & 0.00 \\
Age group $>$ 64 years & -0.24 & 0.14 & 0.09 \\
Forecasting horizon $<$ 16 years & 0.22 & 0.09 & 0.01 \\
N. children & 0.00 & 0.01 & 0.97 \\
N. parents alive & 0.13 & 0.03 & 0.00 \\
N.diseases & -0.05 & 0.02 & 0.05 \\
\hline Self-assessed health & & & \\
Very good & -0.15 & 0.08 & 0.06 \\
Good & -0.53 & 0.08 & 0.00 \\
Fair & -0.90 & 0.09 & 0.00 \\
Poor & -1.40 & 0.12 & 0.00 \\
\hline Marital status & & & \\
Never Married/Annulled & 0.02 & 0.07 & 0.74 \\
Separated/Divorced & 0.05 & 0.05 & 0.31 \\
Widowed & 0.09 & 0.10 & 0.37 \\
\hline Job status & & & \\
Unemployed and looking for a job & 0.15 & 0.09 & 0.08 \\
Temporarily laid off, on sick or other leave & -0.10 & 0.16 & 0.53 \\
Disabled & -0.09 & 0.07 & 0.20 \\
Retired & -0.04 & 0.10 & 0.04 \\
Homemaker & -0.21 & 0.10 & 0.04 \\
Other job status & 0.04 & 0.21 & 0.87 \\
\hline Life satisfaction & & & \\
Very satisfied & 0.01 & 0.06 & 0.88 \\
Somewhat satisfied & -0.14 & 0.06 & 0.03 \\
Not very/not at all satisfied & -0.24 & 0.10 & 0.01 \\
\hline Observations & 9,724 & & \\
\hline & & & \\
\hline
\end{tabular}

Table 9: The table reports regressions results for subjective survival probabilities. The logit of the subjective survival probability is the dependent variable. Gender, age, self-assessed health, life satisfaction, marital status, and job status are categorical variables. The respective reference categories are males, aged between 50 and 64 , self-assessing their health as excellent, extremely satisfied about their life, married, and currently employed.

and 2016, unless we refer to life satisfaction which is reported starting in 2008 only. We consider individuals participating to at least two consecutive waves of the study, which we call "time 0" and "time 1", respectively. The time span between two subsequent waves is two or three years, so we compare probabilities ${ }_{h} p_{x}^{s u b j}(t)$ and ${ }_{h-l} p_{x}^{s u b j}(t+l)$, where $l=2$ or 3 years, with fixed target age according to Table 4 , and analyse their differences $\Delta p_{x}^{s u b j}=$ ${ }_{h} p_{x}^{s u b j}(t){ }_{h-l} p_{x}^{\text {subj }}(t+l)$. We also compute the actuarial counterparts ${ }_{h} p_{x}^{a c t}(t),{ }_{h-l} p_{x}^{a c t}(t+l)$, and $\Delta p_{x}^{a c t}={ }_{h} p_{x}^{a c t}(t)-{ }_{h-l} p_{x}^{a c t}(t+l)$, respectively.

We select all individuals who are healthy at time 0 and consider four different scenarios depending on the health status at time 1:

(1) the individual is again healthy at time $1(H H)$;

(2) one moderately severe impairment (high blood pressure, emotional disorder) is diagnosed between time 0 and time $1\left(H U 1^{a}\right)$;

(3) one severe impairment (diabetes, cancer, heart condition) is diagnosed between time 
(4) the onset of two impairments occurs between time 0 and time 1 (HU2).

In Table 10 we report average changes in subjective probabilities $\left(\Delta p_{x}^{s u b j}\right)$ and in their actuarial counterparts $\left(\Delta p_{x}^{\text {subj }}\right)$. For individuals aged between 50 and 64, we also computed changes in the conditional actuarial probabilities to survive age 80 given that age 75 is reached, i.e., $\Delta p_{x}^{a c t, c o n d}={ }_{r \mid s} p_{x}^{a c t}(t){ }_{r-l \mid s-l} p_{x}^{a c t}(t+l)$, where $r=75-x$ and $s=80-x$ and ${ }_{r \mid s} p_{x}^{a c t}$ is given by Equation (3). We tested if changes in probabilities are significantly different from 0 using a Welch's t-test and all $p$-values are shown in brackets. The main observation is that individuals tend not to change their survival expectations between two subsequent waves. This contrast with the actuarial assessment, because actuarial probabilities either increase $\left(H H\right.$ and $\left.H U 1^{a}\right)$ or decrease $\left(H U 1^{b}\right.$ and $\left.H U 2\right)$, and all changes are significantly different from 0 . In the scenarios healthy-healthy $(H H)$ and healthy-moderatediseases $\left(H U 1^{a}\right)$ actuarial probabilities correctly account for the fact that the target age is approaching, and thus survival at time 1 refers to a shorter horizon. By contrast, in the case of 1 or 2 severe diseases being diagnosed at time 1, the actuarial survival probabilities decrease by $6-7 \%$ for younger individuals and by $9-10 \%$ for older individuals.

Under-reaction to new information (i.e., the appearance of severe diseases) is particularly strong among the elderly population, as predicted by our model. By contrast, younger individuals tend to be closer to actuarial probabilities. Nevertheless, in their most probable scenario healthy-healthy $(H H, 85.56 \%$ of individuals belong to this case) their subjective assessment significantly decreases between two subsequent waves, leading to a negative gap with actuarial probabilities. Overall, the empirical findings support our hypothesis that subjective survival probabilities under-react to new information. Moreover, younger individuals over-react to information that is less relevant for survival. All described results are robust with respect to the exclusion of focal answers 0, 50 and 100 (see Kleinjans and Van Soest 2014 for a discussion on the issue of "reporting behavior").

Next we check if individuals account for existing diseases when they express their own assessment of their health status. Our goal is to verify if the reported under-reaction to health shocks in assessing survival probabilities could be explained by the fact that individuals do not change their view of their health after a shock occurs. We analyse average changes in self-assessed health $(S A H)$ between two subsequent waves (time 0 and time 1 ) in each of the four scenarios previously considered. Table 11 shows our results based on all waves of the HRS study from 2008 to 2016 for which we also have the reported life satisfaction ratings. We observe that self-assessed health, expressed according to a rating 


\begin{tabular}{|c|c|c|c|c|c|c|c|c|c|}
\hline & \multicolumn{6}{|c|}{ Age $(x)$ between 50 and 64} & \multicolumn{3}{|c|}{ Age $(x)$ between 65 and 90} \\
\hline & \multicolumn{3}{|c|}{ Target age 75} & \multicolumn{3}{|c|}{ Target age 80} & & & \\
\hline & & $\Delta p_{x}^{\text {subj }}$ & $\overline{\Delta p_{x}^{a c t}}$ & $\Delta p_{x}^{\text {subj }}$ & $\overline{\Delta p_{x}^{a c t}}$ & $\overline{\Delta p_{x}^{a c t, c o n d}}$ & & $\overline{\Delta p_{x}^{s u b j}}$ & $\overline{\Delta p_{x}^{a c t}}$ \\
\hline$H H$ & 85.56 & $\begin{array}{l}-0.68 \\
(0.00)\end{array}$ & $\begin{array}{c}1.46 \\
(0.00)\end{array}$ & $\begin{array}{l}-0.41 \\
(0.07)\end{array}$ & $\begin{array}{c}1.30 \\
(0.00)\end{array}$ & $\begin{array}{l}-0.08 \\
(0.00)\end{array}$ & 80.25 & $\begin{array}{c}0.65 \\
(0.06)\end{array}$ & $\begin{array}{r}3.47 \\
(0.00)\end{array}$ \\
\hline$H U 1^{a}$ & 3.94 & $\begin{array}{l}-0.71 \\
(0.28)\end{array}$ & $\begin{array}{c}0.36 \\
(0.00)\end{array}$ & $\begin{array}{l}-0.52 \\
(0.49)\end{array}$ & $\begin{array}{c}0.08 \\
(0.00)\end{array}$ & $\begin{array}{l}-0.24 \\
(0.00)\end{array}$ & 6.59 & $\begin{array}{c}0.08 \\
(0.94)\end{array}$ & $\begin{array}{l}1.45 \\
(0.00)\end{array}$ \\
\hline$H U 1^{b}$ & 8.72 & $\begin{array}{l}-1.76 \\
(0.09)\end{array}$ & $\begin{array}{l}-6.35 \\
(0.00)\end{array}$ & $\begin{array}{l}-1.83 \\
(0.10)\end{array}$ & $\begin{array}{l}-8.17 \\
(0.00)\end{array}$ & $\begin{array}{l}-1.89 \\
(0.00)\end{array}$ & 10.54 & $\begin{array}{c}0.77 \\
(0.57)\end{array}$ & $\begin{array}{l}-9.41 \\
(0.00)\end{array}$ \\
\hline HU2 & 1.78 & $\begin{array}{l}-1.94 \\
(0.23)\end{array}$ & $\begin{array}{l}-7.43 \\
(0.00)\end{array}$ & $\begin{array}{c}0.33 \\
(0.84)\end{array}$ & $\begin{array}{l}-9.53 \\
(0.00)\end{array}$ & $\begin{array}{l}-2.15 \\
(0.00)\end{array}$ & 2.62 & $\begin{array}{l}-3.61 \\
(0.09)\end{array}$ & $\begin{array}{l}-10.38 \\
(0.00)\end{array}$ \\
\hline
\end{tabular}

Table 10: The table reports average changes of subjective survival probabilities $\Delta p_{x}^{s u b j}=$ ${ }_{h} p_{x}^{s u b j}(t){ }_{h-l} p_{x}^{s u b j}(t+l)$ and of their actuarial counterparts $\Delta p_{x}^{a c t}={ }_{h} p_{x}^{a c t}(t)-{ }_{h-l} p_{x}^{a c t}(t+l)$ between two subsequent waves distanced by $l \in\{2,3\}$ years, called period 0 and 1 , respectively. All reported changes are percentages. For individuals aged between 50 and 64 in period 0 we consider target ages 75 (columns 2 and 3) and 80 (columns 4 and ) years old, i.e., $h=75-x$ and $80-x$, respectively. For individuals aged 65 and more (columns 7 and 8 ) in period 0 we take fixed target ages between the two subsequent waves according to Table 4. For individuals aged between 50 and 64 in period 0 and when the target age is 80 , we also report the average change of the actuarial conditional probability that an individual survives 80 given that she survives 75 , i.e., $\Delta p_{x}^{a c t, \text { cond }}={ }_{r \mid s} p_{x}^{a c t}(t){ }_{r-l \mid s-l} p_{x}^{a c t}(t+l)$, where $r=75-x$ and $s=80-x$ and ${ }_{r \mid s} p_{x}^{a c t}$ is computed according to Equation (3) (column 6). We study different scenarios concerning the individuals' health status in period 1 . In period 0 all individuals are reported to be healthy $(H)$. In period 1 they are either healthy again (scenario $\mathrm{HH}$ ), or diagnosed with a moderate disease (high blut pressure, emotional disorder, scenario $H U 1^{a}$ ), or with a severe disease (diabetes, cancer, hearth condition, scenario $H U 1^{b}$ ), or with 2 diseases (scenario $H U 2$ ). We applied a Welch's t-test to test the hypothesis that reported differences equal 0. $p$-values are reported in brackets.

from 1 (excellent) to 5 (poor), tend to account for existing diseases. Indeed, overall $S A H$ is lower (i.e., the rating increases) after a disease has been diagnosed between time 0 and time 1 . We also observe that $S A H$ deteriorates when a serious disease or more diseases are diagnosed compared to the case where a moderate disease is diagnosed. However, we also observe that younger individuals similarly change their assessment of their health status after a moderate or a severe disease is diagnosed, while older individuals similarly react to one severe disease or two diseases. Overall, $S A H$ is not insensitive to health shocks, so individuals generally change their self-assessment of the health status after an health shock realizes. Therefore, the fact that subjective probabilities tend to stay similar after an health shock is observed can be hardly explained by referring to individuals completely misjudging their health status.

We also analyse how reported life satisfaction $(L S)$ changes between two subsequent waves, again considering the four different scenarios analysed so far. Interestingly, LS is insensitive with respect to diagnosed diseases. Combining this result with the previously reported evidence showing that $L S$ is generally high, we conclude that individuals tend to optimistically think about their life despite diagnosed with one or two, possible severe, diseases. Our result also shows that younger individuals slightly decrease their life sat- 
isfaction after observing a moderate health shock. Despite the change is marginal (the rating increases by 0.07 on average) it is significantly different from zero and consistent with the hypothesis that younger individuals over-react to health shocks with marginal impact on survival. This is also reflected into their subjective survival probabilities, which remain unchanged despite the actuarial counterparts slightly increase due to the target age approaching. This pattern is also consistent with our theoretical prediction of Section II.

\begin{tabular}{ccccc}
\hline & \multicolumn{2}{c}{ Age $(x)$ in $[50,64]$} & Age $(x)$ & in $\frac{[65,90]}{\Delta L S}$ \\
\cline { 2 - 5 } & $\Delta S A H$ & $\frac{\Delta L A H}{\Delta L S}$ & 0.07 & 0.00 \\
\hline$H H$ & 0.05 & -0.04 & $(0.00)$ & $(0.94)$ \\
\hline$H U 1^{a}$ & 0.26 & 0.07 & 0.24 & 0.00 \\
& $(0.00)$ & $(0.04)$ & $(0.00)$ & $(0.94)$ \\
\hline$H U 1^{b}$ & 0.27 & -0.01 & 0.46 & 0.05 \\
& $(0.00)$ & $(0.83)$ & $(0.00)$ & $(0.41)$ \\
\hline$H U 2$ & 0.42 & 0.11 & 0.43 & 0.12 \\
& $(0.00)$ & $(0.22)$ & $(0.00)$ & $(0.22)$ \\
\hline
\end{tabular}

Table 11: The table reports average changes in self-assessed health $S A H$ and life satisfaction $L S$ between two subsequent waves distanced by two or three years, called time 0 and time 1 , respectively. We separately analyse individuals aged between 50 and 64 in period 0 (columns 2 and 3 ) and individuals aged 65 or more in period 0 (columns 4 and 5). We study different scenarios concerning the individuals' health status in period 1 . In period 0 all considered individuals are reported to be healthy $(H)$. In period 1 they are either healthy again (scenario $H H$ ), or diagnosed with a moderate disease (high blood pressure, emotional disorder, scenario $H U 1^{a}$ ), or with a severe disease (diabetes, cancer, hearth condition, scenario $H U 1^{b}$ ), or with 2 diseases (scenario $H U 2$ ). We applied a Welch's t-test to test the hypothesis that reported differences equal 0. $p$-values are reported in brackets.

To further corroborate our hypothesis that sentiment plays an important role for the dynamics of subjective probabilities, we study which factors drives the changes in subjective and actuarial probabilities between two subsequent waves of the HRS study. Formally, we run OLS regressions for $\Delta p_{x}^{s u b j}, \Delta p_{x}^{a c t}$, and for the difference-of-differences (diff-of-diff) $\Delta p_{x}^{\text {subj }}-\Delta p_{x}^{a c t}$. Here $\Delta p_{x}^{\text {subj }}={ }_{h} p_{x}^{\text {subj }}(t){ }_{{ }_{h-l}} p_{x}^{\text {subj }}(t+l)$ and $\Delta p_{x}^{a c t}={ }_{h} p_{x}^{a c t}(t)-{ }_{h-l} p_{x}^{a c t}(t+l)$ are again the changes in subjective and actuarial survival probabilities, respectively, between two subsequent waves, called time 0 and time 1 , distanced by $l=2$ or 3 years. We consider all individuals who are healthy at time 0 and study the impact of none, one, or several health shocks on their updating behaviour. We separately analyse male and female participants to account for the different dynamics of actuarial probabilities in the two groups. Table 12 reports all our results.

First of all, consistently with our previous results (see Table 10), both female and male respondents under-react to diagnosed diseases. Indeed, the difference in the number of diseases between time 0 and time 1 does not play any significant role for the subjective updates, while it is highly significant for actuarial probabilities, as any additional diag- 
nosed disease reduces the actuarial probability of surviving by $4.79 \%$ for females and by $6.02 \%$ for males. Second, our regression also confirms that individuals tend to not sufficiently increase their subjective expectations when the target age is approaching, while they tend to not sufficiently decrease their expectations when the forecasting horizon and the target age become higher. This is captured by the estimated coefficient for the change in the forecasting horizon. For younger individuals (less than 62 years old) the target age is fixed, and thus the change in the forecasting horizon between two subsequent waves is always negative. Therefore, the highly significant coefficient for this variable implies a positive effect on $\Delta p_{x}$, for both the subjective and the actuarial estimates. However, in the case of the subjective probabilities the coefficient is significantly higher than for the actuarial counterpart ( $-1.69 \%$ vs $-3.14 \%$ for females and $1.67 \%$ vs $-3.34 \%$ for males), which implies that despite individuals account for the target age approaching they do so much less than the actuarial assessment does, leading to an under-estimation of survival probabilities when no disease is diagnosed. By contrast, for older individuals the target age increases by five years every five years (see Table 4). Therefore, between two subsequent waves distanced by 2 or 3 years, the forecasting horizon either decreases (if the individual stays within the same 5-year interval with fixed target age) or increases by 2 or 3 years (if the individual moves into the next 5-year interval with an higher target age). On average older individuals experience an increase of their forecasting horizon, i.e., the change of the forecasting horizon is positive on average. Therefore, the negative coefficients for this variable imply the opposite effect as for younger individuals, i.e., survival probabilities (both subjective and actuarial) decrease due to a longer forecasting horizon. However, for both females and males this effect is much smaller for subjective probabilities compared to their actuarial counterparts, leading to an over-estimation of survival expectations due to not properly taking into account the higher target age.

Next we consider self-assessed health. It significantly affects subjective probabilities for both female and male participants, i.e., if individuals' self-assessed health deteriorates by one point (the rating increases by one point), subjective expectations decrease by $2.46 \%$ for females and by $2.62 \%$ for males. As we previously discussed, self-assessed health captures the occurrence of diseases, and individuals accordingly adjust their subjective estimation. However, the magnitude of this adjustment is small and only marginally correlated to how actuarial probabilities change. This result further supports the hypothesis that the dynamics of subjective probabilities do contain an objective component, but either individuals do not properly understand the severity of a diagnosed disease, or they assess their health based on partially different criteria than those driving actuarial estimates. Overall, 
their assessment is too optimistic and leads to an overestimation of survival probabilities, consistently with our prediction.

Finally, we study the role of life satisfaction which is assumed to correlate with sentiment, as previously discussed. Reported life satisfaction affects subjective survival expectations of female respondents who are not very or not at all satisfied with their life. In this case the impact is statistically significant and high, as those individuals decrease their subjective probabilities by $4.87 \%$ compared to completely satisfied individuals and this result holds after controlling for self-assessed health, the occurrence of diseases, and an increase in the forecasting horizon. This finding further supports our hypothesis that subjective probabilities are affected by sentiment. Indeed, we also report that life satisfaction is not correlated to actuarial probabilities, consistently with our previously reported results showing that life satisfaction tends to remain unchanged after the occurrence of a possibly severe disease. In relation to life satisfaction, we also see a difference due to gender, as male participants' subjective probabilities do not seem to be related to poor life satisfaction, indicating that males tend to optimistically update their probabilities.

Overall the empirical evidence is consistent with the prediction of our model that subjective beliefs are driven by sentiment and that individuals are generally optimistic. We observe that younger individuals tend to over-react to health shocks with marginal impact on survival. Because this scenario is the most frequent one for younger individuals, overall we obtain an underestimation of subjective probabilities. By contrast, older individuals tend to under-react to shocks with a significant impact on survival. This behaviour leads to an overestimation of survival probabilities.

\section{Conclusion}

Residual lifetime is an uncertain outcome on which individuals form subjective beliefs, with implications on the economic decisions they make. Existing literature documents a gap between survival subjective beliefs and actuarial estimates, consisting in survival underestimation at young ages and overestimation at older ages. Despite a broad consensus

on the existence of a survival gap, it is still subject of discussion whether such discrepancy is due to psychological biases.

In this paper, we propose a novel approach to study the role of behavioural biases in relation to longevity risk. First, we develop a model of sentiment in which optimistic and pessimistic expectations about the future health status co-exist. In such a theoretical framework individuals update their subjective assessment of the prevailing sentiment 

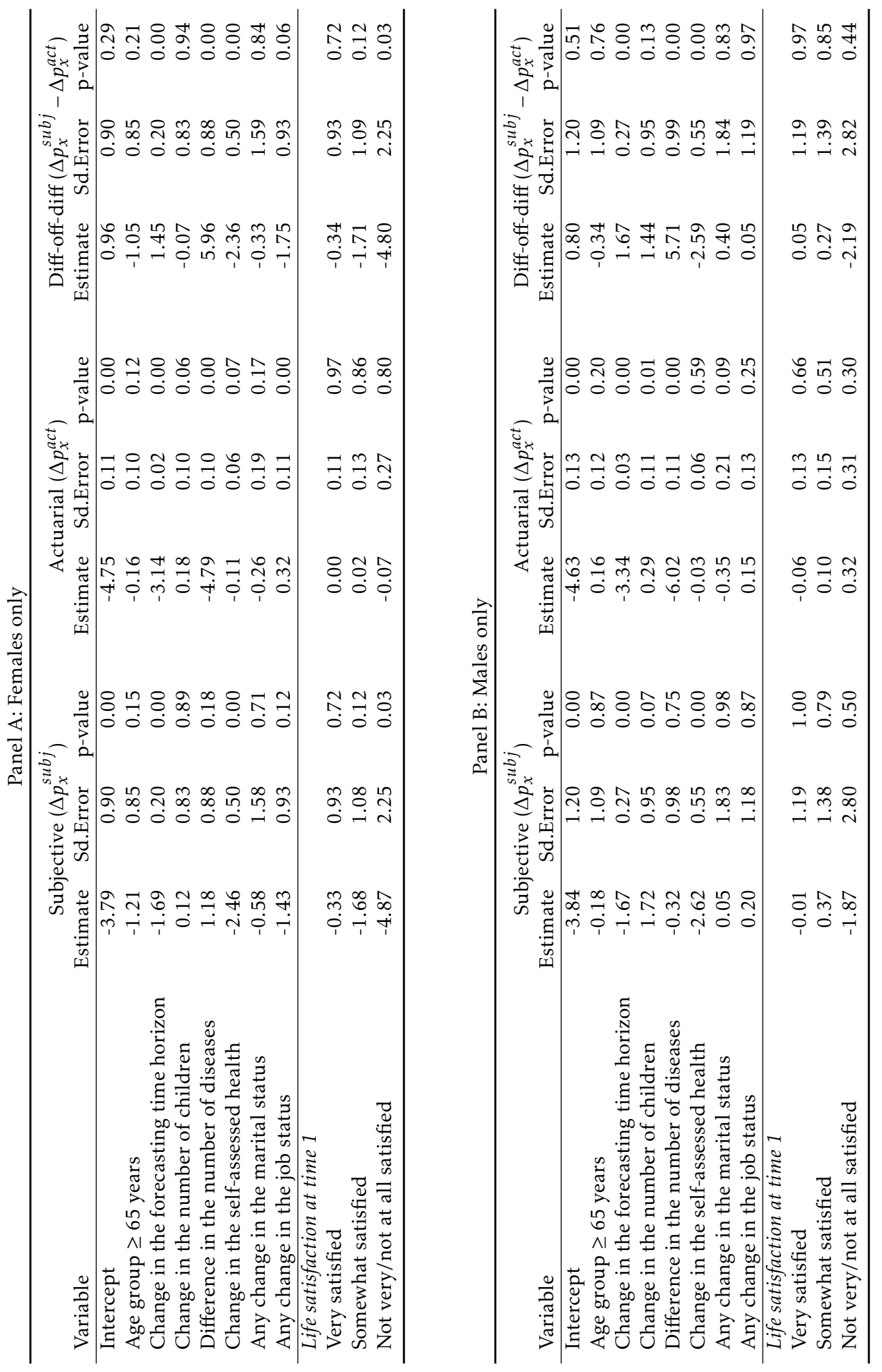

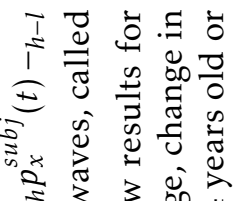

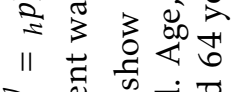

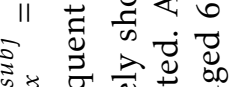

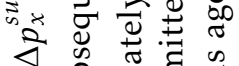

$\checkmark \frac{\pi}{3}$ 范

के के

उे 30.

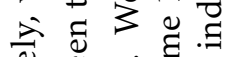

:

过

के 1 च

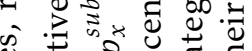

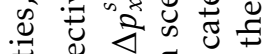

$\because$ के

సิ

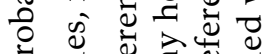

ప.

. 즈 군

ब त प त्व

ป

व

उ $\overline{2} \cong \pm$

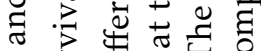

¿ के चें

式

สำ

क उ

पै

¿

र ब क्ष

$\checkmark D 0 \stackrel{0}{0}$

ฮี

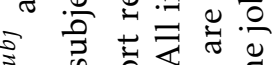

का

$<$ ¿ $₫ . \Xi$

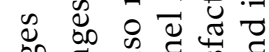

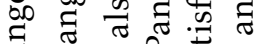

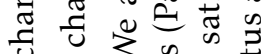

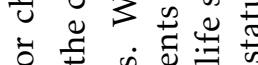

0

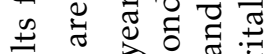

$\overrightarrow{3}=\hat{\alpha}$ की

$+\infty \stackrel{0}{0}$

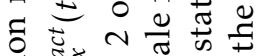

क 2 II $0 . \Xi$

क I - - .0

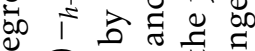

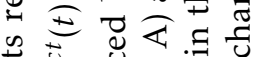

吼

वे इ चै व

\% 11 :

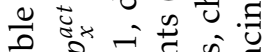

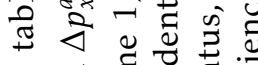

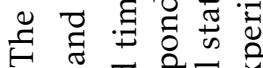

$\ddot{\nu} \stackrel{\sigma}{=} \stackrel{0}{\infty} \approx$

$\sim \pm 0$ 元

교

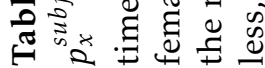


based on their experienced health shocks, this leading to over-reaction or under-reaction to such events. Indeed, our model jointly predicts underestimation of survival beliefs at younger ages and overestimation at older ages assuming that individuals' optimism tend to persist over time, consistently with psychological research. Our model also implies that the more extreme the individual's sentiment prior, the larger the magnitude of survival under- and over-estimation. Second, we validate our models' predictions by exploiting longitudinal data from the U.S. Health and Retirement Study (HRS), along with mortality data from the Human Mortality Database (HMD). With respect to their healthdependent actuarial counterparts, subjective survival probabilities are lower (higher) for younger (older) individuals and decrease more slowly as a function of age. This is consistent with our model of sentiment when individuals are generally optimistic. Moreover, we show that this pattern is related to individual's sentiment.

Existing literature already stresses that individuals' subjective assessment of their own survival affects economic behaviour and that, with respect to some crucial forward-looking decisions (e.g. savings and retirement), subjective beliefs are a contributor of the deviation of actual behaviour from the one predicted by standard economic theories. Nevertheless, whereas the problem (i.e. survival gap) has been clearly detected, the literature only partially addresses the investigation of its nature and of its determinants. This paper gives a relevant contribution in this sense, since we demonstrate that subjective beliefs about survival are driven by sentiment more than by private information. Furthermore, we disentangle the objective component of subjective beliefs from the more emotional one, playing the major role.

The following step in the research process is to exploit our behavioural framework and findings for the improvement of individuals' decision-making. Indeed, the behavioural implications of the survival gap represent a widely open issue. For instance, a behavioural perspective could be very effective in supporting the design of insurance products, e.g. annuities and life insurance, in such a way to nudge individuals to insure themselves against longevity risk. By way of an example, pricing schemes can be adapted to account for the shift from survival pessimism at younger ages to survival optimism at older ages. A similar flexible design would have a quantifiable impact on the attractiveness of the products themselves, net of the biases that may affect potential policyholders at the time when they decide how and how much they should allocate for their post-retirement consumption. 


\section{References}

Anderson, R. N. and H. M. Rosenberg (1998). "Age Standardization of Death Rates: Implementation of the Year 2000 Standard". In: National Vital Statistics Reports 47.3. Hyattsville, Maryland: National Center for Health Statistics.

Atkinson, David B. and John K. McGarry (2016). "Experience Study Calculations". In: Society of Actuaries.

Bailey, Thomas C. et al. (2007). "Hope and optimism as related to life satisfaction". In: The Journal of Positive Psychology 2.3, pp. 168-175. eprint: https : / / doi . org/ 10 . 1080 / 17439760701409546.

Barberis, Nicholas, Andrei Shleifer, and Robert Vishny (1998). "A model of investor sentiment". In: Journal of Financial Economics 49.3, pp. 307-343.

Benartzi, Shlomo, Alessandro Previtero, and Richard H. Thaler (2011). "Annuitization Puzzles". In: Journal of Economic Perspectives 25.4, pp. 143-64.

Benartzi, Shlomo and Richard H. Thaler (1995). "Myopic Loss Aversion and the Equity Premium Puzzle”. In: Quarterly Journal of Economics 110.1, pp. 75-92.

Benyamini, Yael (2011). "Why does self-rated health predict mortality? An update on current knowledge and a research agenda for psychologists". In: Psychology \& Health 26.11, pp. 1407-1413.

Bernheim, B. Douglas (1991). "How Strong Are Bequest Motives? Evidence Based on Estimates of the Demand for Life Insurance and Annuities". In: Journal of Political Economy 99.5, pp. 899-927.

Bernheim, B. Douglas et al. (Oct. 1999). The Adequacy of Life Insurance: Evidence from the Health and Retirement Survey. NBER Working Papers 7372. National Bureau of Economic Research, Inc.

- (2003). "The Mismatch Between Life Insurance Holdings and Financial Vulnerabilities: Evidence from the Health and Retirement Study". In: American Economic Review 93.1, pp. 354-365.

Bjuggren, Carl Magnus and Niklas Elert (2019). "Gender differences in optimism”. In: Applied Economics 51.47, pp. 5160-5173. eprint: https:// doi .org/10 . 1080/00036846. 2019. 1610714.

Blanchflower, David G. (2020). "Is happiness U-shaped everywhere? Age and subjective well-being in 145 countries". In: Journal of Population Economics.

Blass, Asher A., Saul Lach, and Charles F. Manski (2010). "Using Elicited Choice Probabilities to Estimate Random Utility Models: Preferences for Electricity Reliability". In: International Economic Review 51.2, pp. 421-440.

Brown, Jeffrey R. (2007a). “3. Are the Elderly Really Over-Annuitized? New Evidence on Life Insurance and Bequests". In: Themes in the Economics of Aging. Ed. by David A. Wise. University of Chicago Press, pp. 91-126.

- (Oct. 2007b). Rational and Behavioral Perspectives on the Role of Annuities in Retirement Planning. NBER Working Papers 13537. National Bureau of Economic Research, Inc.

Brown, Jeffrey R. et al. (2008). "Why Don't People Insure Late-Life Consumption? A Framing Explanation of the Under-Annuitization Puzzle". In: American Economic Review 98.2, pp. 304-09. 
Bryant, F. B. and J. A. Cvengros (2004). "Distinguishing hope and optimism: Two sides of a coin, or two separate coins?" In: Journal of Social and Clinical Psychology 23.2, pp. 273302.

Cairns, Andrew J. G., David Blake, and Kevin Dowd (2006). "Pricing Death: Frameworks for the Valuation and Securitization of Mortality Risk". In: Astin Bulletin 36.1, pp. 79120.

Cairns, Andrew J. G. et al. (2009). "A Quantitative Comparison of Stochastic Mortality Models using Data from England and Wales and the United States". In: North American Actuarial Journal 13.1, pp. 1-35.

Chopik, W. J., E. S. Kim, and J. Smith (2015). “Changes in Optimism Are Associated With Changes in Health Over Time Among Older Adults". In: Social Psychological and Personality Science 6.7, pp. 814-822.

Chopik, William J. et al. (2020). "Changes in optimism and pessimism in response to life events: Evidence from three large panel studies". In: Journal of Research in Personality 88, p. 103985.

Cummins, J. D. et al. (1983). Risk Classification in Life Insurance. Vol. 1. Huebner International Series on Risk, Insurance, and Economic Security book series. Springer Science+Business Media, LLC.

Cummins, Robert and Helen Nistico (2002). "Maintaining Life Satisfaction: The Role of Positive Cognitive Bias”. In: Journal of Happiness Studies 3.1, pp. 37-69.

Cummins, Robert A. (1998). "The Second Approximation to an International Standard for Life Satisfaction". In: Social Indicators Research 43.3, pp. 307-334.

Cutler, David and Richard Zeckhauser (Jan. 2004). "Extending the Theory to Meet the Practice of Insurance". In: Brookings Wharton Paper Financ Services 2004, pp. 1-53.

Davidoff, T., J. R. Brown, and P. A. Diamond (2005). "Annuities and Individual Welfare". In: American Economic Review 95, pp. 1573-1590.

Elder, Todd E. (2013). "The Predictive Validity of Subjective Mortality Expectations: Evidence From the Health and Retirement Study". In: Demography 50, pp. 569-589.

Forfar, D. O., J. J. McCutcheon, and A. D. Wilkie (1988). "On graduation by mathematical formula". In: Journal of the Institute od Actuaries (1886-1994) 115.1, pp. 1-149.

Greenwood, Robin and Andrei Shleifer (2014). "Expectations of Returns and Expected Returns". In: Review of Financial Studies 27.3, pp. 714-746.

Grevenbrock, Nils et al. (2021). "Cognition, Optimism and the Formation of Age-Dependent Survival Beliefs". In: International Economic Review. Forthcoming. eprint: https : / / onlinelibrary.wiley.com/doi/pdf/10.1111/iere.12497.

Grover, Natalie (2020). “Q\&A: Why some people's wellbeing has improved during the pandemic". In: Horizon. The EU Research E Innovation Magazine.

Haberman, Steven and Arthur Renshaw (2011). "A comparative study of parametric mortality projection models". In: Insurance: Mathematics and Economics 48.1, pp. 35-55.

Hamermesh, Daniel S. (1985). "Expectations, Life Expectancy, and Economic Behavior". In: The Quarterly Journal of Economics 100.2, pp. 389-408.

Hartley, Daniel, Anna L. Paulson, and Katerina Powers (2017). "What Explains the Decline in Life Insurance Ownership?” In: Economic Perspectives 8, pp. 1-20. 
Heimer, Rawley Z., Kristian Ove R. Myrseth, and Raphael S. Schoenle (2019). "YOLO: Mortality Beliefs and Household Finance Puzzles". In: The Journal of Finance 74.6, pp. 2957-2996. eprint: https : / / onlinelibrary . wiley . com / doi / pdf / 10 . 1111 / jofi. 12828.

Human Mortality Database (2020). "University of California, Berkeley (USA), and Max Planck Institute for Demographic Research (Germany)". In: http : //www . mortality . org or http: // www. humanmortality. de. (data downloaded on January 25, 2020).

Hunter, Arthur (1932). "Selection of risks for life insurance". In: Journal of the Institute of Actuaries (1886-1994) 63.2, pp. 173-217.

Hurd, M. D. and K. McGarry (2002). “The Predictive Validity of Subjective Probabilities of Survival”. In: The Economic Journal 112.482, pp. 966-985.

Inkmann, Joachim, Paula Lopes, and Alexander Michaelides (2011). "How Deep Is the Annuity Market Participation Puzzle?” In: The Review of Financial Studies 24.1, pp. 279319.

Jacobsen, B. et al. (2014). "Gender differences in optimism and asset allocation”. In: Journal of Economic Behavior E Organization 107.Part B, pp. 630-651.

Joshanloo, Mohsen and Veljko Jovanović (2020). "The relationship between gender and life satisfaction: analysis across demographic groups and global regions". In: Archives of women's mental health 23.3, 331-338.

Kahneman, D. and A. Tversky (1979). "Prospect Theory: An Analysis of Decisions under Risk”. In: Econometrica 47.2, pp. 263-291.

Kim, Kyoung Tae et al. (Mar. 2020). "The Decrease in Life Insurance Ownership: Implications for Financial Planning”. In: Financial Services Review 28, pp. 1-16.

Kleinjans, Kristin J. and Arthur Van Soest (2014). "Rounding, focal point answers and nonresponse to subjective probability questions". In: Journal of Applied Econometrics 29.4, pp. 567-585.

Kunreuther, Howard C. and Mark V. Pauly (2004). "Neglecting Disaster: Why Don't People Insure Against Large Losses?” In: Journal of Risk and Uncertainty 28.1, pp. 5-21.

Laursen, Thomas M., Merete Nordentoft, and Preben B. Mortensen (2013). "Excess early mortality in schizophrenia". In: Annual review of clinical psychology 10, pp. 425-448.

Lee, R. D. and L. R. Carter (1992). "Modelling and forecasting U.S. mortality". In: Journal of the American Statistical Association 87.14, pp. 659-675.

Liebman, Jeffrey and Richard Zeckhauser (Sept. 2008). Simple Humans, Complex Insurance, Subtle Subsidies. NBER Working Papers 14330. National Bureau of Economic Research, Inc.

Ludwig, A. and A. Zimper (2013). "A parsimonious model of subjective life expectancy". In: Theory and Decision 75.4, pp. 519-541.

Manski, C. (2004). "Measuring Expectations". In: Econometrica 72, pp. 1329-1376.

Manski, C. and F. Molinari (2010). "Rounding Probabilistic Expectaions in Surveys". In: Journal of Business and Economic Statistics 28, pp. 219-231.

Mavros, George et al. (2017). "Stochastic Mortality Modeling: Key Drivers and Dependent Residuals". In: North American Actuarial Journal 21.3, pp. 343-368. 
O' Dea, Corman and David Sturrock (2020). "Survival Pessimism and the Demand for Annuities". In: NBER Working Paper 27677.

Organization for Economic Cooperation and Development (2019). Global Insurance Market Trends.

Pavot, William and Ed Diener (2007). "The Satisfaction With Life Scale and the emerging construct of life satisfaction". In: The Journal of Positive Psychology 3.2, pp. 137-152.

Perozek, Maria (2008). "Using Subjective Expectations to Forecast Longevity: Do Survey Respondents Know Something We Don't Know?" In: Demography 45.1, pp. 95-113.

Piper, Alan (2019). Optimism, pessimism and life satisfaction: an empirical investigation. Discussion Papers 030. Europa-Universität Flensburg, International Institute of Management.

Pitacco, E. et al. (2009). Modelling Longevity Dynamics for Pensions and Annuity Business. Oxford University Press, ISBN:9780199547272.

Pitacco, Ermanno (2010). Matematica e tecnica attuariale delle assicurazioni sulla durata di vita. Lint.

Post, Thomas and Katja Hanewald (2013). "Longevity risk, subjective survival expectations, and individual saving behavior". In: Journal of Economic Behavior Organization 86, pp. 200-220.

Previtero, Alessandro (2014). "Stock market returns and annuitization". In: Journal of Financial Economics 113.2, pp. 202-214.

Puri, Manju and David T. Robinson (2007). "Optimism and economic choice". In: Journal of Financial Economics 86.1, pp. 71-99.

Sawadogo, Relwendé, Samuel Guérineau, and Idrissa Ouedrago (June 2018). "Life insurance development and economic growth: Evidence from developing countries". In: Journal of Economic Development 43, pp. 1-28.

Smith, V. Kerry, Donald H. Taylor, and Frank A. Sloan (2001). "Longevity Expectations and Death: Can People Predict Their Own Demise?" In: The American Economic Review 91.4, pp. 1126-1134.

Wu, Shang, Ralph Stevens, and Susan Thorp (2015). "Cohort and target age effects on subjective survival probabilities: Implications for models of the retirement phase". In: Journal of Economic Dynamics \& Control 55, pp. 39-56.

Wurm, Susanne, Martin J. Tomasik, and Clemens Tesch-Römer (2009). "Serious health events and their impact on changes in subjective health and life satisfaction: the role of age and a positive view on ageing". In: European Journal of Ageing 5.2, pp. 117-127.

$\mathrm{Xu}$, Mengyi, Michael Sherris, and Ramona Meyricke (2019). "Systematic Mortality Improvement Trends and Mortality Heterogeneity: Insights from Individual-Level HRS Data". In: North American Actuarial Journal, pp. 1-23.

Yaari, M. E. (1965). "Uncertain Lifetime, Life Insurance, and the Theory of the Consumer". In: The Review of Economic Studies 32.2, pp. 137-150.

Zimper, Alexander (2012). "Asset pricing in a Lucas fruit-tree economy with the best and worst in mind". In: Journal of Economic Dynamics and Control 36.4, pp. 610-628. 


\section{Appendix}

\section{A Proof of Lemma 1.}

We have:

$$
\begin{aligned}
& \sigma_{x+1}\left(y_{x+1}, y_{x}, \sigma_{x}\right)=\mathbb{P}\left[s_{x+1}=1 \mid y_{x+1}, y_{x}, \sigma_{x}\right] \\
& \quad=\frac{\mathbb{P}\left[s_{x+1}=1, y_{x+1} \mid y_{x}, \sigma_{x}\right]}{\mathbb{P}\left[y_{x+1} \mid y_{x}, \sigma_{x}\right]} \\
& =\frac{\mathbb{P}\left[y_{x+1} \mid s_{x+1}=1, y_{x}, \sigma_{x}\right] \mathbb{P}\left[s_{x+1}=1 \mid y_{x}, \sigma_{x}\right]}{\sum_{k=1}^{2} \mathbb{P}\left[y_{x+1} \mid s_{x+1}=k, y_{x}, \sigma_{x}\right] \mathbb{P}\left[s_{x+1}=k \mid y_{x}, \sigma_{x}\right]} \\
& =\frac{\mathbb{P}\left[y_{x+1} \mid s_{x+1}=1, y_{x}, \sigma_{x}\right]\left[\left(1-\lambda_{1}\right) \sigma_{x}+\lambda_{2}\left(1-\sigma_{x}\right)\right]}{\mathbb{P}\left[y_{x+1}, y_{x}, \sigma_{x}\right]} \\
& =\frac{\mathbb{P}\left[y_{x+1} \mid s_{x+1}=1, y_{x}, \sigma_{x}\right]\left[\left(1-\lambda_{1}\right) \sigma_{x}+\lambda_{2}\left(1-\sigma_{x}\right)\right]}{\sum_{k=1}^{2} \mathbb{P}\left[y_{x+1} \mid s_{x+1}=k, y_{x}, \sigma_{x}\right] \mathbb{P}\left[s_{x+1}=k \mid y_{x}, \sigma_{x}\right]} \\
& =\frac{\mathbb{P}\left[y_{x+1} \mid s_{x+1}=1, y_{x}, \sigma_{x}\right]\left[\left(1-\lambda_{1}\right) \sigma_{x}+\lambda_{2}\left(1-\sigma_{x}\right)\right]}{\mathbb{P}\left[y_{x+1} \mid s_{x+1}=1, y_{x}, \sigma_{x}\right]\left[\left(1-\lambda_{1}\right) \sigma_{x}+\lambda_{2}\left(1-\sigma_{x}\right)\right]+\mathbb{P}\left[y_{x+1} \mid s_{x+1}=2, y_{x}, \sigma_{x}\right]\left[\lambda_{1} \sigma_{x}+\left(1-\lambda_{2}\right)\left(1-\sigma_{x}\right)\right]},
\end{aligned}
$$

where

$$
\mathbb{P}\left[y_{x+1} \mid s_{x+1}=1, y_{x}, \sigma_{x}\right]= \begin{cases}\pi_{1 L} & \text { if } y_{x+1}=y_{x}=0, \\ \pi_{1 H} & \text { if } y_{x+1}=y_{x}=y, \\ 1-\pi_{1 L} & \text { if } y_{x+1}=y \text { and } y_{x}=0, \\ 1-\pi_{1 H} & \text { if } y_{x+1}=0 \text { and } y_{x}=y .\end{cases}
$$

and

$$
\mathbb{P}\left[y_{x+1} \mid s_{x+1}=2, y_{x}, \sigma_{x}\right]= \begin{cases}\pi_{2 H} & \text { if } y_{x+1}=y_{x}=0, \\ \pi_{2 L} & \text { if } y_{x+1}=y_{x}=y, \\ 1-\pi_{2 H} & \text { if } y_{x+1}=y \text { and } y_{x}=0, \\ 1-\pi_{2 L} & \text { if } y_{x+1}=0 \text { and } y_{x}=y .\end{cases}
$$




\section{B Proof of Lemma 2.}

We have:

$$
\begin{gathered}
{ }_{h} p_{x}^{\text {subj }}\left(\eta_{x}, y_{x}, y_{x-1}, \sigma_{x}\right)=\mathbb{P}\left[\eta_{x+h}>\eta^{\star} \mid \eta_{x}, y_{x}, y_{x-1}, \sigma_{x}\right] \\
=\sum_{y_{x+1}} \mathbb{P}\left[\eta_{x+h}>\eta^{\star} \mid y_{x+1}, \eta_{x}, y_{x}, y_{x-1}, \sigma_{x}\right] \mathbb{P}\left[y_{x+1} \mid \eta_{x}, y_{x}, y_{x-1}, \sigma_{x}\right] \\
=\sum_{y_{x+1}} \mathbb{P}\left[\eta_{x+h}>\eta^{\star} \mid y_{x+1}, \eta_{x}, y_{x}, y_{x-1}, \sigma_{x}\right]\left[\sum_{k=1}^{2} \mathbb{P}\left[y_{x+1} \mid s_{x+1}=k, y_{x}, \sigma_{x}\right] \mathbb{P}\left[s_{x+1}=k \mid y_{x}, \sigma_{x}\right]\right] \\
=\sum_{y_{x+1}}^{h-1} p_{x+1}^{s u b j}\left(\eta_{x}+y_{x+1}, y_{x+1}, y_{x}, \sigma_{x+1}\right)\left[\sum_{k=1}^{2} \mathbb{P}\left[y_{x+1} \mid s_{x+1}=k, y_{x}, \sigma_{x}\right] \mathbb{P}\left[s_{x+1}=k \mid y_{x}, \sigma_{x}\right]\right] \\
=\sum_{y_{x+1}}^{h-1} p_{x+1}^{s u b j}\left(\eta_{x}+y_{x+1}, y_{x+1}, y_{x}, \sigma_{x+1}\right) \\
\quad \times\left\{\mathbb{P}\left[y_{x+1} \mid s_{x+1}=1, y_{x}, \sigma_{x}\right]\left[\left(1-\lambda_{1}\right) \sigma_{x}+\lambda_{2}\left(1-\sigma_{x}\right)\right]\right. \\
\left.+\mathbb{P}\left[y_{x+1} \mid s_{x+1}=2, y_{x}, \sigma_{x}\right]\left[\lambda_{1} \sigma_{x}+\left(1-\lambda_{2}\right)\left(1-\sigma_{x}\right)\right]\right\}
\end{gathered}
$$

for $x=0,1, \ldots, x+h-1$ and

$$
{ }_{0} p_{x+h}^{s u b j}\left(\eta_{x+h}, y_{x+h}, y_{x+h-1}, \sigma_{x+h}\right)= \begin{cases}1 & \text { if } \eta_{x+h}>\eta^{\star} \\ 0 & \text { if } \eta_{x+h}<\eta^{\star}\end{cases}
$$

